\title{
On the Sum-Capacity of Degraded Gaussian Multiple-Access Relay Channels
}

\author{
Lalitha Sankar, Member, IEEE, Narayan B. Mandayam, Fellow, IEEE, and H. Vincent Poor, Fellow, IEEE
}

\begin{abstract}
The sum-capacity is studied for a $K$-user physically degraded Gaussian multiple-access relay channel (MARC). Decode-and-forward (DF) is shown to achieve the sum-capacity and capacity region for a subclass of degraded Gaussian MARCs in which the multiple-access link from the sources to the relay is the bottleneck link. For the remaining subclass, DF is shown to achieve the $K$-user sum-capacity when the sources are symmetric, i.e., they transmit with the same transmit power. The optimality of DF is conjectured for the case of asymmetric sources.
\end{abstract}

Index Terms-Gaussian and degraded Gaussian MARC, multiple-access relay channel (MARC), polymatroids, sum-capacity.

\section{INTRODUCTION}

$\mathbf{T}$ HE multiple-access relay channel (MARC) is a network in which several users (sources) communicate with a single destination in the presence of a relay [1]. The coding strategies developed for the relay channel [2], [3] extend readily to the MARC [4], [5]. For example, the strategy of [3, Theorem 1], now often called decode-and-forward (DF), has a relay that decodes user messages before forwarding them to the destination [4], [5]. Similarly, the strategy in [3, Theorem 6], now often called compress-and-forward (CF), has the relay quantize its output symbols and transmit the resulting quantized bits to the destination [5].

Capacity results for relay channels are known only for a few special cases such as the class of degraded relay channels [3] and its multirelay generalization [6], [7], the class of semi-deterministic relay channels [8], the class of orthogonal relay channels [9], [10], the class of Gaussian relay without delay channels [11], [12], and the class of ergodic phase-fading relay channels [4].

For the class of physically degraded relay channels, the degradedness condition requires that the received signal at the destination be independent of the source signal when conditioned on the transmit and receive signals at the relay. For the Gaussian case, this simplifies to the requirement that the signal received at

\footnotetext{
Manuscript received April 02, 2008; revised August 07, 2009. Current version published November 20, 2009. The work of L. Sankar and N. B. Mandayam was supported in part by the National Science Foundation under Grant ITR-0205362. The work of H. V. Poor was supported by the National Science Foundation under Grants ANI-03-38807 and CNS-06-25637. The material in this paper was presented in part at the Information Theory and Applications Workshop, La Jolla, CA, January 2007.

L. Sankar and H. V. Poor are with the Department of Electrical Engineering, Princeton University, Princeton, NJ 08544 USA (e-mail: lalitha@ princeton.edu; poor@princeton.edu).

N. B. Mandayam is with WINLAB, Rutgers University, Technology Center of NJ, North Brunswick, NJ 08902 USA (e-mail: narayan@winlab.rutgers.edu). Communicated by H. Yamamoto, Associate Editor for Shannon Theory.

Color versions of Figures 1-3 in this paper are available online at http://ieeexplore.ieee.org.

Digital Object Identifier 10.1109/TIT.2009.2032821
}

the destination be a sum of the signal at the relay and an independent additive white Gaussian noise component, conditioned on the transmitted signal at the relay. This condition immediately suggests that requiring the relay to decode the source signals should be optimal. In fact, for this class, applying this degradedness condition simplifies the cut-set outer bounds to coincide with the DF bounds.

We define a $K$-user physically degraded (henceforth referred to simply as degraded) Gaussian MARC as one where the multiple-access signal received at the destination from the $K$ sources and relay is a sum of the received signal at the relay from all sources and an independent additive white Gaussian noise signal, conditioned on the transmit signal at the relay. For this channel, we develop the DF rate region as an inner bound on the capacity region using Gaussian signaling at the sources and relay.

We obtain outer bounds on the capacity region of the degraded Gaussian MARC by specializing the cut-set bounds of [13, Theorem 14.10.1] to the case of independent sources [14] and by applying the degradedness condition. Applying the physically degradedness condition to the cutset outer bounds, however, does not simplify the bounds to those achieved by DF. In fact, the inner and outer bounds differ in their input distributions as well as the rate bounds.

Our motivation in developing the $K$-user sum-capacity for this channel stems from the observation that if the physically degraded destination can decode the signals from all sources, as required of it, so can the relay, i.e., DF must be sum-capacity optimal. To this end, we first show the optimality of Gaussian signaling in the outer bounds. This in turn allows us to show that both the inner (DF) and the outer bounds on the $K$-user sum-rate are functions of the $K$ correlation coefficients, one for each source-relay signal pair. In fact, for appropriate choice of the DF correlation coefficients, the inner and outer bounds on the $K$-user sum-rate (and only that) can be shown to be the same.

This simplification, however, does not imply that DF is sumcapacity optimal. This is due to the fact that for any choice of input and output distributions, the inner and outer bound rate regions are given by an intersection of two polymatroids, one resulting from the multiple-access region at the relay and the other from the multiple-access region at the destination.

Applying a single-known result in matroid theory on the intersection of two polymatroids [15, Ch. 46], we show that the intersection of two polymatroids can be classified as either an active or an inactive case when the constraints on $K$-user sum-rate planes at the two receivers are active or not active in the final intersection, respectively. Thus, we show that the sum-rate for the 
active cases is a minimum of the two $K$-user sum-rates while that for an inactive case is a sum of the rate-sums achieved by a subset of users and its complement at the relay and destination, respectively. Thus, for an active case, the inner and outer bounds on the $K$-user sum-rate are the same. However, unlike the $K$-user sum-rates, the outer bounds on the rate-sum for any subset of users is at least as large as the inner bounds, and thus, for an inactive case, the maximum inner and outer bound sum-rate may not be the same.

Despite this, applying polymatroid theory allows us to broadly classify the class of degraded Gaussian MARCs into one of two disjoint subclasses. The first subclass is one in which the relay-to-destination channel is a high-capacity link such that the $K$-user multiple-access link from the sources to the relay becomes a bottleneck link. For this subclass, we show that DF achieves the sum-capacity and the capacity region when each source allocates all its power to transmitting its signal to the relay, and thus, does not allocate any power to cooperating with the relay to achieve coherent combining gains at the destination.

The second subclass is the one in which the $K$-user multipleaccess link from the sources to the relay is not a bottleneck link as a result of which one or more sources cooperate with the relay to enhance their sum-rate at the destination. For this subclass, we can show that DF is optimal only if the source and relay correlation coefficients that maximize the $K$-user DF sum-rate result in a polymatroid intersection that is an active case. While we can precisely determine the sum-rate maximizing source and relay correlation coefficients, referred to as max-min rules, it is not straightforward to verify that there exists a max-min rule that results in an active case.

For the second subclass, we show that DF is optimal when the source powers are the same, i.e., for a symmetric degraded Gaussian MARC in which the $K$-user multiple-access link from the sources to the relay is not a bottleneck link. The symmetry allows us to prove that there exists a max-min rule that results in an active case. Finally, we exploit the symmetric solution to argue and conjecture that DF is sum-capacity optimal for any channel belonging to the second subclass.

This paper is organized as follows. In Section II, we present a model for a degraded Gaussian MARC and briefly overview polymatroids and their intersections. We summarize our main results in Section III and prove them in Sections IV, V, and VI. We conclude in Section VII.

\section{Channel MOdEl AND PRELIMINARIES}

A $K$-user degraded Gaussian MARC has $K$ user (source) nodes, one relay node, and one destination node (see Fig. 1). The sources emit the messages $W_{k}, k=1,2, \ldots, K$, which are statistically independent and take on values uniformly in the sets $\left\{1,2, \ldots, M_{k}\right\}$. The channel is used $n$ times so that the rate of $W_{k}$ is $R_{k}=B_{k} / n$ bits per channel use where $B_{k}=\log _{2} M_{k}$ bits. In each use of the channel, the input to the channel from source $k$ is $X_{k}$ while the relay's input is $X_{r}$. The channel outputs $Y_{r}$ and $Y_{d}$, respectively, at the relay and the destination are

$$
Y_{r}=\left(\sum_{k=1}^{K} X_{k}\right)+Z_{r}
$$

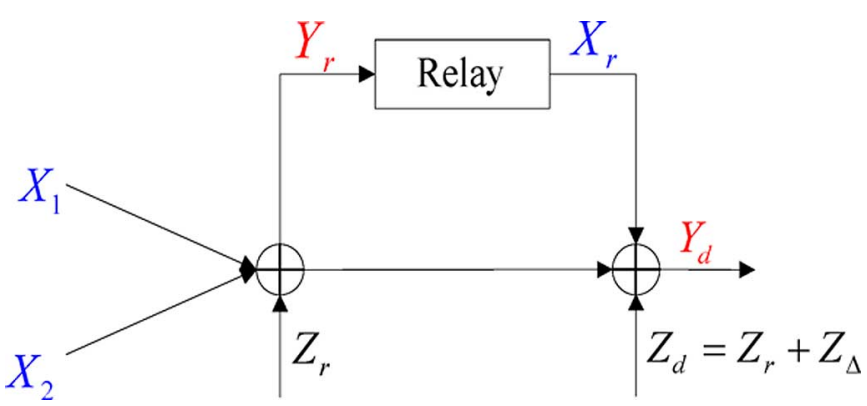

Fig. 1. A two-user Gaussian degraded MARC.

$$
\begin{aligned}
Y_{d} & =\left(\sum_{k=1}^{K} X_{k}\right)+X_{r}+Z_{d} \\
& =Y_{r}+X_{r}+Z_{\Delta}
\end{aligned}
$$

where $Z_{r}$ and $Z_{\Delta}$ are independent Gaussian random variables with zero means and variances $N_{r}$ and $N_{\Delta}$, respectively, such that the noise variance at the destination is

$$
N_{d}=N_{r}+N_{\Delta}
$$

We assume that the relay operates in a full-duplex manner, i.e., it can transmit and receive simultaneously in the same bandwidth. Further, its input $X_{r}$ in each channel use is a causal function of its outputs from previous channel uses. We write $\mathcal{K}=\{1,2, \ldots, K\}$ for the set of sources, $\mathcal{T}=\mathcal{K} \cup\{r\}$ for the set of transmitters, $\mathcal{R}=\{r, d\}$ for the set of receivers, $X_{\mathcal{S}}=\left\{X_{k}: k \in \mathcal{S}\right\}$ for all $\mathcal{S} \subseteq \mathcal{K}$, and $\mathcal{S}^{c}$ to denote the complement of $\mathcal{S}$ in $\mathcal{K}$.

The transmitted signals from source $k$ and the relay have a per-symbol power constraint

$$
E\left[\left|X_{k}\right|^{2}\right] \leq P_{k}, \quad k \in \mathcal{T}
$$

One can equivalently express the relationship between the input and output signals in (3) as a Markov chain

$$
\left(X_{1}, X_{2}, \ldots, X_{K}\right)-\left(Y_{r}, X_{r}\right)-Y_{d}
$$

For $K=1$, (6) simplifies to the degradedness condition in [3, eq. (10)] for the classic (single-source) relay channel. A degraded Gaussian MARC is symmetric if $P_{k}=P$, for all $k$. Thus, the class of symmetric degraded Gaussian MARCs is characterized by four parameters, namely, $P, P_{r}, N_{r}$, and $N_{d}$.

The capacity region $\mathcal{C}_{\text {MARC }}$ is the closure of the set of rate tuples $\left(R_{1}, R_{2}, \ldots, R_{K}\right)$ for which the destination can, for sufficiently large $n$, decode the $K$ source messages with an arbitrarily small positive error probability. As further notation, we write $R_{\mathcal{S}}=\sum_{k \in \mathcal{S}} R_{k}$. We write $\underline{0}$ and $\underline{1}$ to denote vectors whose entries are all zero and one, respectively, and $C(x)=\log (1+x) / 2$ to denote the capacity of an additive white Gaussian noise channel with signal-to-noise ratio (SNR) $x$. We use the usual notation for entropy and mutual information [13], [16] and take all logarithms to the base 2 so that in each channel use our rate units are bits. 


\section{A. Polymatroids}

In the sequel, we use the properties of polymatroids to develop the inner and outer bounds on the $K$-user sum-rate. Polymatroids have been used to develop capacity characterizations for a variety of multiple-access channel models including the MARC (see, for example, [17]-[19]). We review the following definition of a polymatroid.

Definition 1: Let $\mathcal{K}=\{1,2, \ldots, K\}$ and $f=2^{K} \rightarrow \mathfrak{R}_{+}$be a set function. The polyhedron

$$
\mathcal{B}(f) \equiv\left\{\left(R_{1}, R_{2}, \ldots, R_{K}\right): 0 \leq R_{\mathcal{S}} \leq f(\mathcal{S}), \text { for all } \mathcal{S} \subseteq \mathcal{K}\right\}
$$

is a polymatroid if $f$ satisfies

1) $f(\emptyset)=0$ (normalization)

2) $f(\mathcal{S}) \leq f(\mathcal{P})$ if $\mathcal{S} \subset \mathcal{P}$ (monotonicity)

3) $f(\mathcal{S})+f(\mathcal{P}) \geq f(\mathcal{S} \cup \mathcal{P})+f(\mathcal{S} \cap \mathcal{P})$ (submodularity).

Remark 1: The submodularity property in Definition 1 above is equivalent to requiring, for all $k_{1}, k_{2}$ in $\mathcal{K}$ with $k_{1} \neq k_{2}$, $k_{1} \notin \mathcal{S}, k_{2} \notin \mathcal{S}$, that $f$ satisfies [15, Ch. 44]

$$
f\left(\mathcal{S} \cup\left\{k_{1}\right\}\right)+f\left(\mathcal{S} \cup\left\{k_{2}\right\}\right) \geq f(\mathcal{S})+f\left(\mathcal{S} \cup\left\{k_{1}, k_{2}\right\}\right) .
$$

This property is used in [20] to show that the rate regions achieved at both the relay and the destination in a full-duplex MARC are polymatroids.

We use the following lemma on polymatroid intersections to develop optimal inner and outer bounds on the sum-rate for $K$-user orthogonal MARCs.

Lemma 1 ([15, p. 796, Corollary 46.1c]): Let $R_{\mathcal{S}} \leq f_{1}(\mathcal{S})$ and $R_{\mathcal{S}} \leq f_{2}(\mathcal{S})$, for all $\mathcal{S} \subseteq \mathcal{K}$, be two polymatroids such that $f_{1}$ and $f_{2}$ are nondecreasing submodular set functions on $\mathcal{K}$ with $f_{1}(\emptyset)=f_{2}(\emptyset)=0$. Then

$$
\max R_{\mathcal{K}}=\min _{\mathcal{S} \subseteq \mathcal{K}}\left(f_{1}(\mathcal{S})+f_{2}(\mathcal{K} \backslash \mathcal{S})\right)
$$

Lemma 1 states that the maximum sum of $R_{k}$, for all $k$, denoted by $R_{\mathcal{K}}$, that results from the intersection of two polymatroids, $R_{\mathcal{S}} \leq f_{1}(\mathcal{S})$ and $R_{\mathcal{S}} \leq f_{2}(\mathcal{S})$, is given by the minimum of the two $K$-variable planes $f_{1}(\mathcal{K})$ and $f_{2}(\mathcal{K})$ only if both sums are at most as large as the sum of the orthogonal planes $f_{1}(\mathcal{S})$ and $f_{2}(\mathcal{K} \backslash \mathcal{S})$, for all $\emptyset \neq \mathcal{S} \subset \mathcal{K}$. We refer to the resulting intersection as an active case.

When there exists at least one $\emptyset \neq \mathcal{S} \subset \mathcal{K}$ for which the above condition is not true, an inactive case is said to result. For such cases, the maximum $K$-variable sum in (7) is the sum of two orthogonal rate planes achieved by two complementary subsets of users. As a result, the $K$-variable sum bounds $f_{1}(\mathcal{K})$ and $f_{2}(\mathcal{K})$ are no longer active for this case, and thus, the region of intersection is no longer a polymatroid with $2^{K}-1$ faces.

An inactive case results when

$f_{1}(\mathcal{S})+f_{2}(\mathcal{K} \backslash \mathcal{S})<\min \left(f_{1}(\mathcal{K}), f_{2}(\mathcal{K})\right)$, for an $\mathcal{S} \subset \mathcal{K}$
Thus, the condition in (8) for an inactive case precludes an active case. Furthermore, the inactive cases are also mutually exclusive. For a $K$-user MARC, there are $2^{K}-2$ possible inactive cases. An active case on the other hand satisfies the condition

$$
f_{1}(\mathcal{S})+f_{2}(\mathcal{K} \backslash \mathcal{S}) \geq \min \left(f_{1}(\mathcal{K}), f_{2}(\mathcal{K})\right), \text { for all } \mathcal{S} \subset \mathcal{K}
$$

In Fig. 2, for two two-dimensional polymatroids, we illustrate the five possible choices for $R_{1}+R_{2}$ from an intersection of $R_{\mathcal{S}} \leq f_{1}(\mathcal{S})$ and $R_{\mathcal{S}} \leq f_{2}(\mathcal{S})$. Cases 1 and 2 are inactive cases while cases $3 a, 3 b$, and $3 c$ are active cases. The sumrate is a minimum of the sum-rates at the two receivers for the active cases $3 a, 3 b$, and $3 c$. For the inactive cases 1 and 2 , the constraints on $R_{1}+R_{2}$ are no longer active and the sum-rate is given by the bounds $f_{1}(\{1\})+f_{2}(\{2\})$ and $f_{1}(\{2\})+f_{2}(\{1\})$, respectively.

\section{MAIN RESULTS}

We summarize our main results in this section. We begin with the outer bounds.

\section{A. Outer Bounds}

In Section IV, for a MARC we present the cut-set outer bounds specialized to the case of independent sources. We further simplify the bounds for the degraded model and show that Gaussian signaling at the sources and relay is optimal for a Gaussian MARC. We define the cross-correlation $\gamma_{k}$ between the Gaussian input signals $X_{k}$ and $X_{r}$ at source $k$ and the relay, respectively, as

$$
E\left[X_{k} X_{r}\right] \triangleq \sqrt{\gamma_{k} P_{k} P_{r}}, \quad \text { for all } k \in \mathcal{K}
$$

such that

$$
\sum_{k \in \mathcal{K}} \gamma_{k} \leq 1
$$

Let $\gamma$ denote the vector of cross-correlation coefficients $\gamma_{k}$, for all $\bar{k} \in \mathcal{K}$ and

$$
\Gamma_{\mathrm{OB}} \triangleq\left\{\underline{\gamma}_{\mathcal{K}}: \sum_{k \in \mathcal{K}} \gamma_{k} \leq 1\right\} .
$$

We write $\underline{\gamma}^{K+1} \equiv\left(\underline{\gamma}^{(1)}, \underline{\gamma}^{(2)}, \ldots, \underline{\gamma}^{(K+1)}\right)$ to denote a collection of $K+1$ cross-correlation vectors and let $\eta$ denote a vector of $K+1$ nonnegative weights $\eta_{m}$ such that

$$
\sum_{m=1}^{K+1} \eta_{m}=1
$$

The following theorem summarizes an outer bound on the capacity region of the degraded Gaussian MARC.

Theorem 1: The capacity region $\mathcal{C}_{\text {MARC }}$ of a degraded Gaussian MARC is contained in the region $\mathcal{R}_{\mathrm{OB}}$ given as

$$
\mathcal{R}_{\mathrm{OB}}=\bigcup_{\sum_{m=1}^{K+1} \eta_{m} \underline{\gamma}^{(m)} \in \Gamma_{\mathrm{OB}}}\left(\mathcal{R}_{r}^{\mathrm{ob}}\left(\underline{\gamma}^{K+1}, \underline{\eta}\right) \cap \mathcal{R}_{d}^{\mathrm{ob}}\left(\underline{\gamma}^{K+1}, \underline{\eta}\right)\right)
$$




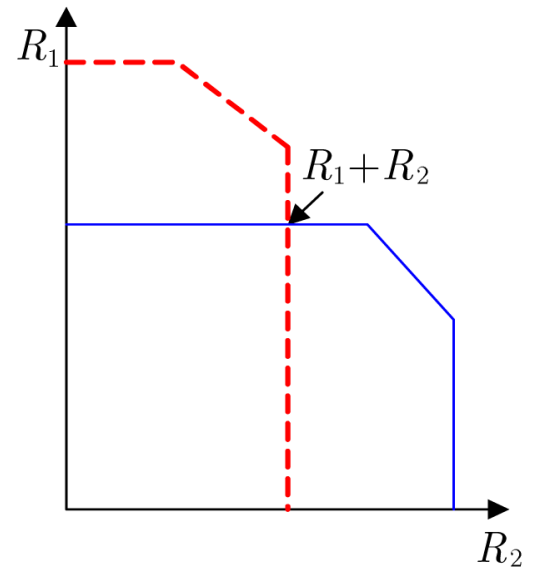

$\underline{\text { Inactive }}$

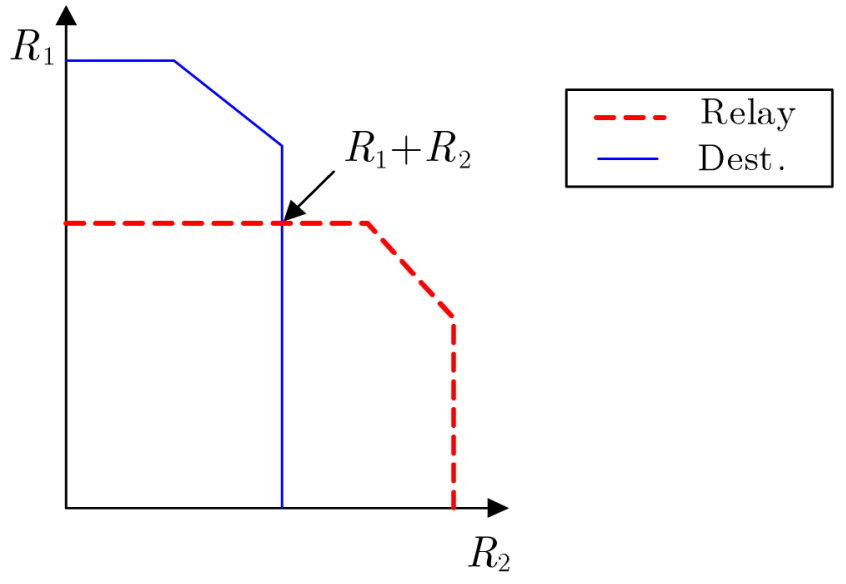

Inactive
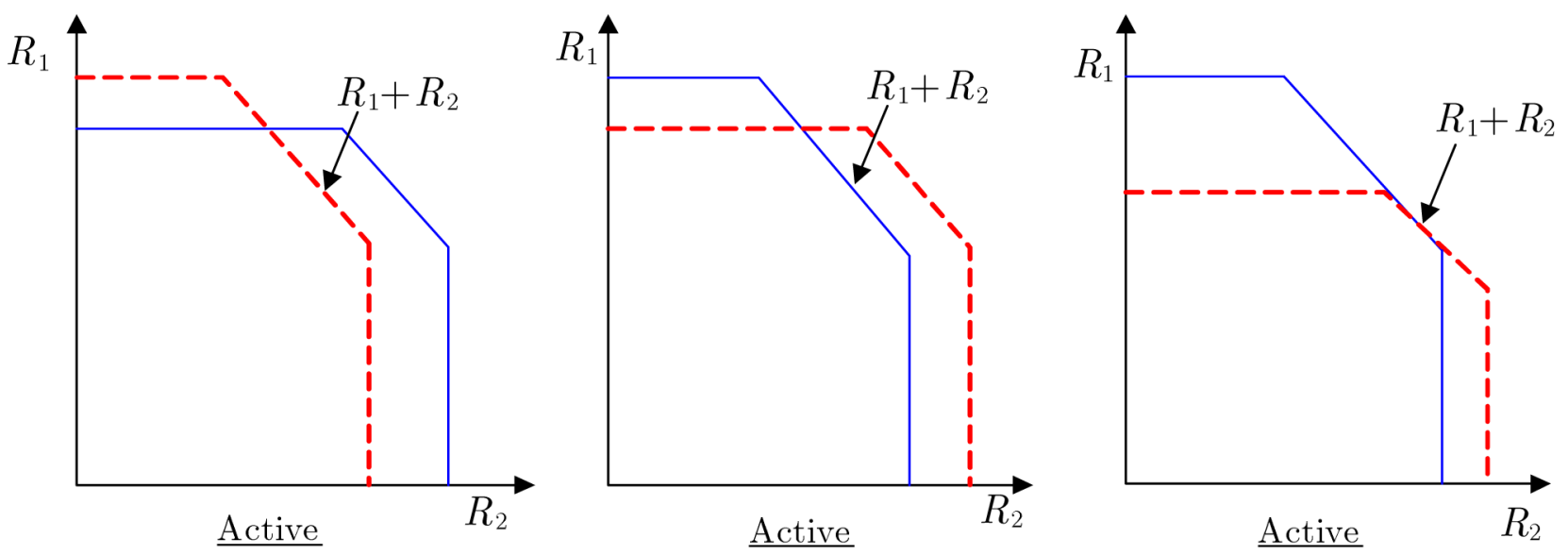

Fig. 2. Five possible intersections of $\mathcal{R}_{r}$ and $\mathcal{R}_{d}$ for a two-user Gaussian MARC.

where the rate regions $\mathcal{R}_{j}^{\mathrm{ob}}\left(\underline{\gamma}^{K+1}, \underline{\eta}\right), j=r, d$, are given by $\mathcal{R}_{j}^{\mathrm{ob}}\left(\underline{\gamma}^{K+1}, \underline{\eta}\right)=$ $\left\{\left(R_{1}, R_{2}, \ldots, R_{K}\right): 0 \leq R_{\mathcal{S}} \leq \sum_{m=1}^{K+1} \eta_{m} B_{j, \mathcal{S}}\left(\underline{\gamma}_{\mathcal{K}}^{(m)}\right)\right\}$.

The bounds $B_{r, \mathcal{S}}\left(\underline{\gamma}_{\mathcal{K}}\right)$ and $B_{d, \mathcal{S}}\left(\underline{\gamma}_{\mathcal{K}}\right)$ are given by

$B_{r, \mathcal{S}}\left(\underline{\gamma}_{\mathcal{K}}\right)= \begin{cases}C\left(\sum_{k \in \mathcal{S}} \frac{P_{k}}{N_{r}}\right), & \text { if } \sum_{k \in \mathcal{S}^{c}} \gamma_{k}=1 \\ C\left(\sum_{k \in \mathcal{S}} \frac{P_{k}}{N_{r}}-\frac{\left.\left(\sum_{k \in \mathcal{S}} \sqrt{\gamma_{k} P_{k}}\right)^{2}\right)}{N_{r} \bar{\gamma}_{\mathcal{S}^{c}}}\right), & \text { otherwise }\end{cases}$

and

$$
B_{d, \mathcal{S}}\left(\underline{\gamma}_{\mathcal{K}}\right)=C\left(\frac{\sum_{k \in \mathcal{S}} P_{k}+\bar{\gamma}_{\mathcal{S}^{c}} P_{r}+2 \sum_{k \in \mathcal{S}} \sqrt{\gamma_{k} P_{k} P_{r}}}{N_{d}}\right)
$$

where

$$
\bar{\gamma}_{\mathcal{S}^{c}}=1-\sum_{k \in \mathcal{S}^{c}} \gamma_{k} .
$$

Remark 2: For $K=1$, the bounds in (16) and (17) simplify to the first and second bound, respectively, for the degraded relay channel in [3, Theorem 5].

Remark 3: In the expression for $B_{d, \mathcal{S}}\left(\underline{\gamma}_{\mathcal{K}}\right)$ in (17), the terms involving the cross-correlation coefficients quantify the coherent combining gains that result from choosing correlated source and relay signals. On the other hand, the expression for $B_{r, \mathcal{S}}\left(\underline{\gamma}_{\mathcal{K}}\right)$ in (16) quantifies the upper bounds on the rate achievable at the relay when one or more source signals are correlated with the transmitted signal at the relay.

\section{B. Inner Bounds}

The DF rate region is obtained using Gaussian signaling at the sources and relay and a block Markov superposition code [4, Appendix A] (see also [20]) . Let $\underline{\alpha}$ and $\beta$ denote vectors with entries $\alpha_{k}$ and $\beta_{k}$, respectively, for all $k \bar{\in} \mathcal{K}$ such that

$$
\Gamma=\left\{\left(\underline{\alpha}_{\mathcal{K}}, \underline{\beta}_{\mathcal{K}}\right): \alpha_{k} \in[0,1], 0 \leq \sum_{k \in \mathcal{K}} \beta_{k} \leq 1\right\} \text {. }
$$


The following theorem summarizes the DF rate region.

Theorem 2: The DF rate region $\mathcal{R}_{\mathrm{DF}}$ for a degraded Gaussian MARC is

$$
\mathcal{R}_{\mathrm{DF}}=\bigcup_{\left(\underline{\alpha}_{\mathcal{K}}, \underline{\beta}_{\mathcal{K}}\right) \in \Gamma}\left(\mathcal{R}_{r}\left(\underline{\alpha}_{\mathcal{K}}\right) \cap \mathcal{R}_{d}\left(\underline{\alpha}_{\mathcal{K}}, \underline{\beta}_{\mathcal{K}}\right)\right)
$$

where the rate region $\mathcal{R}_{t}, t=r, d$, is

$$
\begin{aligned}
\mathcal{R}_{t}\left(\underline{\alpha}_{\mathcal{K}}, \underline{\beta}_{\mathcal{K}}\right) & =\left\{\left(R_{1}, R_{2}, \ldots, R_{K}\right):\right. \\
0 & \left.\leq R_{\mathcal{S}} \leq I_{t, \mathcal{S}}\left(\underline{\alpha}_{\mathcal{K}}, \underline{\beta}_{\mathcal{K}}\right), \text { for all } \mathcal{S} \subseteq \mathcal{K}\right\} .
\end{aligned}
$$

The rate bounds $I_{r, \mathcal{S}}$ and $I_{d, \mathcal{S}}$ at the relay and destination, respectively, are

$$
\begin{aligned}
I_{r, \mathcal{S}}\left(\underline{\alpha}_{\mathcal{K}}\right)=C\left(\frac{\sum_{k \in \mathcal{S}} \alpha_{k} P_{k}}{N_{r}}\right) \text { and } \\
I_{d, \mathcal{S}}\left(\underline{\alpha}_{\mathcal{K}}, \underline{\beta}_{\mathcal{K}}\right)=C\left(\frac{\sum_{k \in \mathcal{S}} P_{k}}{N_{d}}+\frac{\left(1-\sum_{k \in \mathcal{S}^{c}} \beta_{k}\right) P_{r}}{N_{d}}\right. \\
\left.\quad+2 \sum_{k \in \mathcal{S}} \sqrt{\left(1-\alpha_{k}\right) \beta_{k} \frac{P_{k}}{N_{d}} \frac{P_{r}}{N_{d}}}\right) .
\end{aligned}
$$

From Theorems 1 and 2, one can verify that the outer and innner bounds on the $K$-user sum-rate are identical by setting $\gamma_{k}=\left(1-\alpha_{k}\right) \beta_{k}$ for all $k$. In the sequel, we show that the DF bounds are concave functions of $\underline{\alpha}_{\mathcal{K}}$ and $\underline{\beta}_{\mathcal{K}}$ for all $\mathcal{S}$, and thus, so are the $K$-user sum-rate outer bounds. Thus, maximizing the $K$-user DF sum-rate bound is equivalent to maximizing the $K$-user sum-rate outer bound. Note that the outer bound rate region in (14) requires time-sharing because with the exception of $B_{r, \mathcal{K}}$, the bounds $B_{r, \mathcal{S}}$ for all $\mathcal{S} \subset \mathcal{K}$ are in general not convex.

The following theorem summarizes our results on the capacity region and the sum-capacity of a $K$-user degraded Gaussian MARC.

Theorem 3: The capacity region $\mathcal{C}_{\mathrm{MARC}}$ of a $K$-user degraded Gaussian MARC is

$$
\begin{aligned}
& \mathcal{C}_{\mathrm{MARC}}=\mathcal{R}_{r}(\underline{1}), \quad \text { if } \frac{\sum_{k \in \mathcal{K}} P_{k}}{N_{r}} \leq \frac{\sum_{k \in \mathcal{K}} P_{k}}{N_{d}}+\frac{P_{r}}{N_{d}} \\
& \mathcal{R}_{\mathrm{DF}} \subset \mathcal{C}_{\mathrm{MARC}} \subset \mathcal{R}_{\mathrm{OB}}, \quad \text { otherwise }
\end{aligned}
$$

where

$$
\begin{array}{r}
\mathcal{R}_{r}(\underline{1})=\left\{\left(R_{1}, R_{2}, \ldots, R_{K}\right): R_{\mathcal{S}} \leq C\left(\sum_{k \in \mathcal{S}} P_{k} / N_{r}\right),\right. \\
\quad \text { for all } \mathcal{S} \subseteq \mathcal{K}\} .
\end{array}
$$

The sum-capacity $C_{\mathcal{K}}$ of a degraded Gaussian MARC is

$$
C_{\mathcal{K}}=C\left(\frac{\sum_{k \in \mathcal{K}} P_{k}}{N_{r}}\right), \quad \text { if } \frac{\sum_{k \in \mathcal{K}} P_{k}}{N_{r}} \leq \frac{\sum_{k \in \mathcal{K}} P_{k}}{N_{d}}+\frac{P_{r}}{N_{d}}
$$

$$
\begin{aligned}
C_{\mathcal{K}}=I^{*}, \quad \text { if } \frac{\sum_{k \in \mathcal{K}} P_{k}}{N_{r}}>\frac{\sum_{k \in \mathcal{K}} P_{k}}{N_{d}}+\frac{P_{r}}{N_{d}}, \\
\quad \text { and } \mathcal{R}_{r}\left(\underline{\alpha}_{\mathcal{K}}^{*}, \underline{\beta}_{\mathcal{K}}^{*}\right) \cap \mathcal{R}_{d}\left(\underline{\alpha}_{\mathcal{K}}^{*}, \underline{\beta}_{\mathcal{K}}^{*}\right)
\end{aligned} \quad \begin{aligned}
& \quad \text { is an active case, and } \\
& C_{\mathcal{K}}<I^{*}, \quad \text { otherwise }
\end{aligned}
$$

where for

$$
\begin{aligned}
\beta_{k}^{*} & =\left\{\begin{array}{ll}
\frac{\left(1-\alpha_{k}^{*}\right) P_{k}}{\sum_{k=1}^{K}\left(1-\alpha_{k}^{*}\right) P_{k}} & \underline{\alpha}_{\mathcal{K}}^{*} \neq \underline{1} \\
0 & \underline{\alpha}_{\mathcal{K}}^{*}=\underline{1}
\end{array} \text { for all } k \in \mathcal{K},\right. \text { and } \\
q^{*} & \equiv \sqrt{\sum_{k \in \mathcal{K}}\left(1-\alpha_{k}^{*}\right) \lambda_{k}}
\end{aligned}
$$

such that $q^{*}$ is the unique solution of $I_{r, \mathcal{K}}\left(\underline{\alpha}_{\mathcal{K}}^{*}\right)=$ $I_{d, \mathcal{K}}\left(\underline{\alpha}_{\mathcal{K}}^{*}, \underline{\beta}_{\mathcal{K}}^{*}\right)$ given by

$$
q^{*}=\frac{-K_{1}+\sqrt{K_{1}^{2}+\left(K_{3}-K_{2}\right) K_{0}}}{K_{0}}
$$

for

$$
\begin{aligned}
& K_{0}=P_{\max } / N_{r}, \quad K_{1}=\sqrt{P_{\max } P_{r}} / N_{d}, \\
& K_{2}=\frac{\sum_{k \in \mathcal{K}} P_{k}}{N_{d}}+\frac{P_{r}}{N_{d}} \text {, and } K_{3}=\frac{\sum_{k \in \mathcal{K}} P_{k}}{N_{r}} \text {. }
\end{aligned}
$$

we have

$$
\begin{aligned}
I_{r, \mathcal{K}}\left(\underline{\alpha}_{\mathcal{K}}^{*}\right)=I_{d, \mathcal{K}}\left(\underline{\alpha}_{\mathcal{K}}^{*}, \underline{\beta}_{\mathcal{K}}^{*}\right)=I^{*} \\
\quad=C\left(\left(\sum_{k \in \mathcal{K}} \frac{P_{k}}{N_{r}}\right)-\frac{\left(q^{*}\right)^{2} P_{\max }}{N_{r}}\right)
\end{aligned}
$$

for

$$
P_{\max } \equiv \max _{k \in \mathcal{K}}\left(P_{k}\right) \text { and } \lambda_{k} \equiv \frac{P_{k}}{P_{\max }}, \quad \text { for all } k \in \mathcal{K} .
$$

For a symmetric degraded Gaussian MARC that satisfies the condition in (26), DF achieves the sum-capacity

$$
\begin{aligned}
& C_{\mathcal{K}}=I^{*}\left(\underline{\alpha}_{\mathcal{K}}^{*}, \underline{\beta}_{\mathcal{K}}^{*}\right), \quad \text { for } \alpha_{k}^{*}=\alpha^{*}=1-\frac{\left(q^{*}\right)^{2}}{K}, \\
& \beta_{k}^{*}=1 / K, \text { for all } k \text {. }
\end{aligned}
$$

Remark 4: In the sequel, for DF, we define the entries $\alpha_{k}$ and $\beta_{k}$ of $\underline{\alpha}_{\mathcal{K}}$ and $\beta_{\mathcal{K}}$, respectively, for all $k$, as power fractions. In fact, $\alpha_{k}$ is the fraction of the total power that source $k$ allocates to transmitting a new message in a block of $n$ channel uses. On the other hand, the entry $\beta_{k}$, for all $k$, is a fraction of its total power that the relay allocates to cooperating with source $k$.

Conjecture 1: DF achieves the sum-capacity of degraded Gaussian MARCs.

\section{OUteR AND INNER Bounds: RATE REgION}

\section{A. Outer Bounds Rate Region}

In this section, we present a proof of Theorem 1 in which an outer bound $\mathcal{R}_{\mathrm{OB}}$ on the capacity region $\mathcal{C}_{\mathrm{MARC}}$ of a degraded Gaussian MARC is presented. To this end, we begin with an 
outer bound on the capacity region of a MARC which is presented in [14] using the cut-set bounds in [13, Theorem 14.10.1] as applied to the case of independent sources. We summarize the bounds below.

Proposition 1: The capacity region $\mathcal{C}_{\mathrm{MARC}}$ is contained in the union of the set of rate tuples $\left(R_{1}, R_{2}, \ldots, R_{K}\right)$ that satisfy, for all $\mathcal{S} \subseteq \mathcal{K}$,

$R_{\mathcal{S}} \leq \min \left\{I\left(X_{\mathcal{S}} ; Y_{r}, Y_{d} \mid X_{\mathcal{S}^{c}}, X_{r}, U\right), I\left(X_{\mathcal{S}}, X_{r} ; Y_{d} \mid X_{\mathcal{S}^{c}}, U\right)\right\}$

where $U$ is a time-sharing random variable and the union is over all distributions that factor as

$$
p(u) \cdot\left(\prod_{k=1}^{K} p\left(x_{k} \mid u\right)\right) \cdot p\left(x_{r} \mid x_{\mathcal{K}}, u\right) \cdot p\left(y_{r}, y_{d} \mid x_{\mathcal{K}}, x_{r}\right) .
$$

Remark 5: Thetime-sharing random variable $U$ ensures that the region in (34) is convex. One can apply Caratheodory's theorem [21] to this $K$-dimensional convex region to bound the cardinality of $U$ as $|\mathcal{U}| \leq K+1$.

Consider the outer bounds in Proposition 1. For a degraded MARC applying the degradness definition in (6) simplifies (34) as

$$
\begin{array}{r}
R_{\mathcal{S}} \leq \min \left\{I\left(X_{\mathcal{S}} ; Y_{r} \mid X_{r} X_{\mathcal{S}^{c}} U\right), I\left(X_{\mathcal{S}} X_{r} ; Y_{d} \mid X_{\mathcal{S}^{c}} U\right)\right\}, \\
\text { for all } \mathcal{S} \subseteq \mathcal{K}
\end{array}
$$

for the same joint distribution in (35).

In Appendix I, we specialize the bounds in (36) for the Gaussian degraded MARC. For a fixed $U$, we show that the outer bounds are maximized for Gaussian signaling at the sources and the relay, i.e., for $X_{k} \sim \mathcal{N}\left(0, P_{k}\right)$, for all $k$, and $X_{r} \sim \mathcal{N}\left(0, P_{r}\right)$. The cross-correlation coefficient between $X_{k}$ and $X_{r}$ is defined as $\gamma_{k}$, for all $k$, in (10). In Appendix I, we show that $\gamma_{k}$, for all $k$, satisfy (11).

For a given set of transmit powers, $P_{k}, k \in \mathcal{T}$, we write $B_{r, \mathcal{S}}\left(\underline{\gamma}_{\mathcal{K}}\right)$ and $B_{d, \mathcal{S}}\left(\underline{\gamma}_{\mathcal{K}}\right)$ to denote the first and second terms, respectively, in (36), for every $\mathcal{S} \subseteq \mathcal{K}$. In Appendix I, we prove that $B_{r, \mathcal{S}}\left(\underline{\gamma}_{\mathcal{K}}\right)$ and $B_{d, \mathcal{S}}\left(\underline{\gamma}_{\mathcal{K}}\right)$ are given by (16) and (17), respectively.

\section{Proof of Theorem 1}

From (12), we see that the set of all correlation vectors $\underline{\gamma}_{\mathcal{K}}$, denoted as $\Gamma_{\mathrm{OB}}$, is a closed convex set. The bound $B_{r, \mathcal{S}}\left(\underline{\gamma}_{\mathcal{K}}\right)$ in (16), in general, is not a concave function of $\underline{\gamma}_{\mathcal{K}}$ for any $\mathcal{S} \subset \mathcal{K}$. In Appendix III, for a fixed $\underline{\gamma}_{\mathcal{S c}}$, we show that $B_{r, \mathcal{S}}\left(\underline{\gamma}_{\mathcal{K}}\right)$ is a concave function of $\underline{\gamma}_{\mathcal{S}}$. Using this we then show that $B_{r, \mathcal{K}}\left(\underline{\gamma}_{\mathcal{K}}\right)$ is a concave function of $\underline{\gamma}_{\mathcal{K}}$. In Appendix II, we show that for all $\mathcal{S} \subset \mathcal{K}, B_{d, \mathcal{S}}\left(\underline{\gamma}_{\mathcal{K}}\right)$ in (17) is a concave function of $\underline{\gamma}_{\mathcal{K}}$.

The rate region $\mathcal{R}_{\mathrm{OB}}$ enclosed by the outer bounds is obtained as follows. From (36), (16), and (17), for any choice of $\underline{\gamma}_{\mathcal{K}}$, the rate region is an intersection of the multiple-access rate regions at the relay and destination, enclosed by the bounds $B_{r, \mathcal{S}}\left(\underline{\gamma}_{\mathcal{K}}\right)$ and $B_{d, \mathcal{S}}\left(\underline{\gamma}_{\mathcal{K}}\right)$, respectively, for all $\mathcal{S}$. Since $B_{r, \mathcal{S}}\left(\underline{\gamma}_{\mathcal{K}}\right)$ is not a concave function of $\underline{\gamma}_{\mathcal{K}}$, the region can be enlarged by considering all possible convex combinations of
$\underline{\gamma}_{\mathcal{K}}$ to obtain $\mathcal{R}_{\mathrm{OB}}$. For the $K$-dimensional convex region $\mathcal{R}_{\mathrm{OB}}$ we apply Caratheodory's theorem [21] to express every rate tuple $\left(R_{1}, R_{2}, \ldots, R_{K}\right)$ in $\mathcal{R}_{\mathrm{OB}}$ as a convex combination of at most $K+1$ rate tuples, where each rate tuple is obtained for a specific choice of $\underline{\gamma}_{\mathcal{K}}$.

Thus, for every choice of a set of $K+1$ cross-correlation vectors denoted as $\underline{\gamma}^{K+1} \equiv\left(\underline{\gamma}_{(1)}, \underline{\gamma}_{(2)}, \ldots, \underline{\gamma}_{(K+1)}\right)$ and a vector $\eta$ of $K+1$ nonnegative weights $\eta_{m}$ satisfying (13), the rate regions $\mathcal{R}_{r}^{\mathrm{ob}}\left(\underline{\gamma}^{K+1}, \underline{\eta}\right)$ and $\mathcal{R}_{d}^{\mathrm{ob}}\left(\underline{\gamma}^{K+1}, \underline{\eta}\right)$ are given by (15). Finally, the region $\mathcal{R}_{\mathrm{OB}}$ is obtained as a union of $\mathcal{R}_{r}^{\mathrm{ob}}\left(\underline{\gamma}^{K+1}, \underline{\eta}\right)$ and $\mathcal{R}_{d}^{\mathrm{ob}}\left(\underline{\gamma}^{K+1}, \underline{\eta}\right)$ over all choices of $\left(\underline{\gamma}^{K+1}, \underline{\eta}\right)$ and is given by (14) in Theorem 1 where we have used the fact that $\Gamma_{\mathrm{OB}}$ is a convex region.

Lemma 2: The regions $\mathcal{R}_{r}^{\mathrm{ob}}\left(\underline{\gamma}^{K+1}, \underline{\eta}\right)$ and $\mathcal{R}_{d}^{\mathrm{ob}}\left(\underline{\gamma}^{K+1}, \underline{\eta}\right)$ are polymatroids.

Proof: In [20], it is shown that for DF, the multiple-access rate regions at the relay and destination are polymatroids. One can use similar techniques to show that the regions $\mathcal{R}_{r}^{\mathrm{ob}}\left(\underline{\gamma}^{K+1}, \underline{\eta}\right)$ and $\mathcal{R}_{d}^{\mathrm{ob}}\left(\gamma^{K+1}, \underline{\eta}\right)$ are polymatroids and we omit it in the interest of space. Note that for the optimal Gaussian input distribution, this implies that $\mathcal{R}_{r}^{\mathrm{ob}}\left(\underline{\gamma}^{K+1}, \underline{\eta}\right)$ and $\mathcal{R}_{d}^{\mathrm{ob}}\left(\gamma^{K+1}, \eta\right)$ are polymatroids for every choice of $\left(\underline{\gamma}^{K+1}, \underline{\eta}\right)$.

The region $\mathcal{R}_{\mathrm{OB}}$ in (14) is a union of the intersections of the regions $\mathcal{R}_{r}^{\mathrm{ob}}\left(\underline{\gamma}_{\mathcal{K}}, \underline{\eta}\right)$ and $\mathcal{R}_{d}^{\mathrm{ob}}\left(\underline{\gamma}_{\mathcal{K}}, \underline{\eta}\right)$, where the union is taken over all convex combinations of $\underline{\gamma}_{\mathcal{K}}$. Since $\mathcal{R}_{\mathrm{OB}}$ is convex, we obtain the boundary of $\mathcal{R}_{\mathrm{OB}}$ by maximizing the weighted sum $\sum_{k \in \mathcal{K}} \mu_{k} R_{k}$ over all $\Gamma_{\mathrm{OB}}$ and for all $\mu_{k}>0$. Specifically, we determine the sum-rate $R_{\mathcal{K}}$ when $\mu_{k}=1$ for all $k$. The convexity of $\mathcal{R}_{\mathrm{OB}}$ also implies that every point on the boundary of $\mathcal{R}_{\mathrm{OB}}$ results from the intersection of the two polymatroids $\mathcal{R}_{r}^{\mathrm{ob}}\left(\underline{\gamma}^{K+1}, \underline{\eta}\right)$ and $\mathcal{R}_{d}^{\mathrm{ob}}\left(\underline{\gamma}^{K+1}, \underline{\eta}\right)$ for some $\left(\underline{\gamma}^{K+1}, \underline{\eta}\right)$.

\section{B. DF Rate Region}

A DF code construction for a discrete memoryless MARC using block Markov encoding and backward decoding is developed in [4, Appendix A] (see also [20]) and we extend it here to the degraded Gaussian MARC. We first summarize the rate region achieved by DF below.

Proposition 2: The DF rate region is the union of the set of rate tuples $\left(R_{1}, R_{2}, \ldots, R_{K}\right)$ that satisfy, for all $\mathcal{S} \subseteq \mathcal{K}$

$R_{\mathcal{S}} \leq \min \left\{I\left(X_{\mathcal{S}} ; Y_{r} \mid X_{\mathcal{S}^{c}} V_{\mathcal{K}} X_{r} U\right), I\left(X_{\mathcal{S}} X_{r} ; Y_{d} \mid X_{\mathcal{S}^{c}} V_{\mathcal{S}^{c}} U\right)\right\}$

where $U$ is a time-sharing random variable, $V_{1}, V_{2}, \ldots, V_{K}$ are auxiliary random variables, and the union is over all distributions that factor as

$$
p(u) \cdot\left(\prod_{k=1}^{K} p\left(v_{k} \mid u\right) p\left(x_{k} \mid v_{k}, u\right)\right) \cdot p\left(x_{r} \mid v_{\mathcal{K}}, u\right) \cdot p\left(y_{r}, y_{d} \mid x_{\mathcal{T}}\right) \text {. }
$$

Proof: See [20].

Remark 6: Thetime-sharing random variable $U$ ensures that the region of Theorem 2 is convex.

Remark 7: The independent auxiliary random variables $V_{k}$, $k=1,2, \ldots, K$, help the sources cooperate with the relay. 


\section{Proof of Theorem 2}

For the degraded Gaussian MARC, we employ the following code construction. We generate zero-mean, unit variance, independent and identically distributed (i.i.d.) Gaussian random variables $V_{k}, V_{k, 0}$, and $V_{r, 0}$, for all $k \in \mathcal{K}$, such that the channel inputs from source $k$ and the relay are

$$
\begin{aligned}
& X_{k}=\sqrt{\alpha_{k} P_{k}} V_{k, 0}+\sqrt{\left(1-\alpha_{k}\right) P_{k}} V_{k}, \quad k \in \mathcal{K} \\
& X_{r}=\sum_{k=1}^{K} \sqrt{\beta_{k} P_{r}} V_{k}+\sqrt{\left(1-\sum_{k=1}^{K} \beta_{k}\right) P_{r} V_{r, 0}}
\end{aligned}
$$

where $\alpha_{k} \in[0,1]$ and $\beta_{k} \in[0,1]$ are power fractions at source $k$ and the relay, respectively, for all $k$. The set $\Gamma$ of all vectors $\left(\underline{\alpha}_{\mathcal{K}}, \underline{\beta}_{\mathcal{K}}\right)$ with entries $\left(\alpha_{k}, \beta_{k}\right)$ for all $k$ is given by (19). Substituting (39) and (40) in (37), for any $\left(\underline{\alpha}_{\mathcal{K}}, \underline{\beta}_{\mathcal{K}}\right) \in \Gamma$, we obtain

$$
R_{\mathcal{S}} \leq \min \left(I_{r, \mathcal{S}}\left(\underline{\alpha}_{\mathcal{K}}\right), I_{d, \mathcal{S}}\left(\underline{\alpha}_{\mathcal{K}}, \underline{\beta}_{\mathcal{K}}\right)\right), \quad \text { for all } \mathcal{S} \subseteq \mathcal{K}
$$

where $I_{r, \mathcal{S}}$ and $I_{d, \mathcal{S}}$, the bounds at the relay and destination respectively, are given by (22) and (23), respectively. From the concavity of the $\log$ function it follows that $I_{r, \mathcal{S}}$, for all $\mathcal{S}$, is a concave function of $\underline{\alpha}_{\mathcal{K}}$. In Appendix II, we show that $I_{d, \mathcal{S}}$ is a concave function of $\underline{\alpha}_{\mathcal{K}}$ and $\underline{\beta}_{\mathcal{K}}$. The DF rate region, $\mathcal{R}_{\mathrm{DF}}$, achieved over all $\left(\underline{\alpha}_{\mathcal{K}}, \underline{\beta}_{\mathcal{K}}\right) \in \Gamma$, where $\Gamma$ is a convex set, is then given by Theorem 2 .

Lemma 3: The rate region $\mathcal{R}_{\mathrm{DF}}$ is convex.

Proof: To show that $\mathcal{R}_{\mathrm{DF}}$ is convex, it suffices to show that $I_{r, \mathcal{S}}$ and $I_{d, \mathcal{S}}$, for all $\mathcal{S}$, are concave functions over the convex set $\Gamma$ of $\left(\underline{\alpha}_{\mathcal{K}}, \underline{\beta}_{\mathcal{K}}\right)$. This is because the concavity of $I_{r, \mathcal{S}}$ and $I_{d, \mathcal{S}}$, for all $\mathcal{S}$, ensures that a convex sum of two or more rate tuples in $\mathcal{R}_{\mathrm{DF}}$, each corresponding to a different value of $\left(\underline{\alpha}_{\mathcal{K}}, \underline{\beta}_{\mathcal{K}}\right)$ tuple, also belongs to $\mathcal{R}_{\mathrm{DF}}$, i.e., satisfies (21) for $t=r, d$.

Lemma 4: The rate regions $\mathcal{R}_{r}$ and $\mathcal{R}_{d}$ are polymatroids.

Proof: See [20, Sec. IV]. For the Gaussian input distribution in (39) and (40), this implies that $\mathcal{R}_{r}(\underline{\alpha})$ and $\mathcal{R}_{d}\left(\underline{\alpha}_{\mathcal{K}}, \underline{\beta}_{\mathcal{K}}\right)$ are polymatroids for every choice of $\left(\underline{\alpha}_{\mathcal{K}}, \underline{\beta}_{\mathcal{K}}\right)$, i.e., $\mathcal{R}_{r}\left(\underline{\alpha}_{\mathcal{K}}\right)$ and $\mathcal{R}_{d}\left(\underline{\alpha}_{\mathcal{K}}, \underline{\beta}_{\mathcal{K}}\right)$ are completely defined by the corner (vertex) points on their dominant $K$-user sum-rate face [15, Ch. 44].

The region $\mathcal{R}_{\mathrm{DF}}$ in (20) is a union of the intersection of the regions $\mathcal{R}_{r}(\underline{\alpha})$ and $\mathcal{R}_{d}\left(\underline{\alpha}_{\mathcal{K}}, \underline{\beta}_{\mathcal{K}}\right)$ achieved at the relay and destination, respectively, where the union is over all $\left(\underline{\alpha}_{\mathcal{K}}, \underline{\beta}_{\mathcal{K}}\right) \in \Gamma$. Since $\mathcal{R}_{\mathrm{DF}}$ is convex, each point on the boundary of $\mathcal{R}_{\mathrm{DF}}$ is obtained by maximizing the weighted sum $\sum_{k \in \mathcal{K}} \mu_{k} R_{k}$ over all $\Gamma$, and for all $\mu_{k}>0$. Specifically, we seek to determine the optimal policy $\left(\underline{\alpha}_{\mathcal{K}}^{*}, \underline{\beta}_{\mathcal{K}}^{*}\right)$ that maximizes the sum-rate $R_{\mathcal{K}}$ when $\mu_{k}=1$ for all $k$. From (20), we see that every point on the boundary of $\mathcal{R}_{\mathrm{DF}}$ results from the intersection of the polymatroids $\mathcal{R}_{r}\left(\underline{\alpha}_{\mathcal{K}}\right)$ and $\mathcal{R}_{d}\left(\underline{\alpha}_{\mathcal{K}}, \underline{\beta}_{\mathcal{K}}\right)$ for some $\left(\underline{\alpha}_{\mathcal{K}}, \underline{\beta}_{\mathcal{K}}\right)$.

\section{V. $K$-USER SUM-RATE: OUTER AND INNER BOUNDS}

In Section IV, we developed inner DF and outer bounds on the rate region of a degraded Gaussian MARC. Comparing the two bounds, we make the following observations.
1) For any choice of $\left(\underline{\gamma}^{K+1}, \underline{\eta}\right)$ and $\left(\underline{\alpha}_{\mathcal{K}}, \underline{\beta}_{\mathcal{K}}\right)$, the rate regions $\mathcal{R}_{r}^{\mathrm{ob}}\left(\underline{\gamma}^{K+1}, \underline{\eta}\right) \cap \mathcal{R}_{d}^{\mathrm{ob}}\left(\underline{\gamma}^{K+1}, \underline{\eta}\right)$ and $\mathcal{R}_{r}(\underline{\alpha}) \cap \mathcal{R}_{d}\left(\underline{\alpha}_{\mathcal{K}}, \underline{\beta}_{\mathcal{K}}\right)$, for the inner and outer bounds, respectively, are obtained from an intersection of two polymatroids. Thus, from Lemma 1 their intersection is either an active case or an inactive case.

2) The functions $B_{r, \mathcal{K}}\left(\underline{\gamma}_{\mathcal{K}}\right)$ and $B_{d, \mathcal{K}}\left(\underline{\gamma}_{\mathcal{K}}\right)$ denoting the outer bounds on the $K$-user sum-rate are concave functions of $\underline{\gamma}_{\mathcal{K}}$. For the inner bounds, the functions $I_{r, \mathcal{K}}\left(\underline{\alpha}_{\mathcal{K}}\right)$ and $\bar{I}_{d, \mathcal{K}}\left(\underline{\alpha}_{\mathcal{K}}, \underline{\beta}_{\mathcal{K}}\right)$ are concave functions of $\left(\underline{\alpha}_{\mathcal{K}}, \underline{\beta}_{\mathcal{K}}\right)$.

Following the observation in 1 ), we can use Lemma 1 to determine the $K$-user sum-rate for both the inner and outer bounds. The following lemma summarizes the maximum $K$-user sumrate given by the outer bounds.

Lemma 5: For each $\left(\underline{\gamma}^{K+1}, \underline{\eta}\right)$ such that $\sum_{m=1}^{K+1} \eta_{m} \underline{\gamma}_{(m)} \in$ $\Gamma_{\text {OB }}$, the maximum $K$-user sum-rate outer bound $R_{\mathcal{K}}^{\text {ob }}\left(\underline{\gamma}_{\mathcal{K}}\right)$ resulting from the intersecting polymatroids $\mathcal{R}_{r}^{\mathrm{ob}}\left(\underline{\gamma}^{K+1}, \underline{\eta}\right)$ and $\mathcal{R}_{d}^{\mathrm{ob}}\left(\underline{\gamma}^{K+1}, \underline{\eta}\right)$ is

$$
R_{\mathcal{K}}^{\mathrm{ob}}\left(\underline{\gamma}_{\mathcal{K}}\right)= \begin{cases}\bar{B}_{d, \mathcal{A}}+\bar{B}_{r, \mathcal{A}^{c}}, & \text { if Condition } 1 \\ \min \left(B_{r, \mathcal{K}}, B_{d, \mathcal{K}}\right), & \text { otherwise }\end{cases}
$$

where Condition 1 in (42) is given by

$$
\begin{aligned}
\text { Condition } 1: \bar{B}_{d, \mathcal{A}}+\bar{B}_{r, \mathcal{A}^{c}}<\min ( & \left(B_{r, \mathcal{K}}, B_{d, \mathcal{K}}\right), \\
& \text { for an } \emptyset \neq \mathcal{A} \subset \mathcal{K} .
\end{aligned}
$$

Remark 8: The condition in (43) is an application of (8) and determines whether the intersection of two polymatroids is an active or an inactive case with respect to the $K$-user sum-rate.

Remark 9: In (42) and (43), we write the $K$-user sum-rate outer bounds as $B_{r, \mathcal{K}}$ and $B_{d, \mathcal{K}}$ due to the fact that $B_{r, \mathcal{K}}$ and $B_{d, \mathcal{K}}$ are concave functions of $\underline{\gamma}$ (see Appendices III and II).

Proof: The proof follows from applying Lemma 1 to the maximization of $R_{\mathcal{K}}$ for each choice of $\left(\underline{\gamma}^{K+1}, \underline{\eta}\right)$.

One can similarly write the expression for the $K$-user DF sum-rate. The following lemma summarizes the expression for the $K$-user sum-rate achieved by DF.

Lemma 6: For any $\left(\underline{\alpha}_{\mathcal{K}}, \underline{\beta}_{\mathcal{K}}\right)$, the maximum $K$-user DF sumrate $R_{\mathcal{K}}^{\mathrm{DF}}\left(\underline{\alpha}_{\mathcal{K}}, \underline{\beta}_{\mathcal{K}}\right)$ resulting from the intersecting polymatroids $\mathcal{R}_{r}$ and $\mathcal{R}_{d}$ is

$$
R_{\mathcal{K}}^{\mathrm{DF}}\left(\underline{\alpha}_{\mathcal{K}}, \underline{\beta}_{\mathcal{K}}\right)= \begin{cases}S_{i}\left(\underline{\alpha}_{\mathcal{K}}, \underline{\beta}_{\mathcal{K}}\right), & \text { if Condition } 2 \\ S_{a}\left(\underline{\alpha}_{\mathcal{K}}, \underline{\beta}_{\mathcal{K}}\right), & \text { otherwise }\end{cases}
$$

where

$$
\begin{aligned}
& S_{i}\left(\underline{\alpha}_{\mathcal{K}}, \underline{\beta}_{\mathcal{K}}\right)=I_{d, \mathcal{A}}\left(\underline{\alpha}_{\mathcal{K}}, \underline{\beta}_{\mathcal{K}}\right)+I_{r, \mathcal{A}}\left(\underline{\alpha}_{\mathcal{K}}, \underline{\beta}_{\mathcal{K}}\right) \\
& S_{a}\left(\underline{\alpha}_{\mathcal{K}}, \underline{\beta}_{\mathcal{K}}\right)=\min \left(I_{r, \mathcal{K}}\left(\underline{\alpha}_{\mathcal{K}}, \underline{\beta}_{\mathcal{K}}\right), I_{d, \mathcal{K}}\left(\underline{\alpha}_{\mathcal{K}}, \underline{\beta}_{\mathcal{K}}\right)\right)
\end{aligned}
$$

and the Condition 2 in (44) is given by

Condition $2: S_{i}\left(\underline{\alpha}_{\mathcal{K}}, \underline{\beta}_{\mathcal{K}}\right)<S_{a}\left(\underline{\alpha}_{\mathcal{K}}, \underline{\beta}_{\mathcal{K}}\right)$, for an $\emptyset \neq \mathcal{A} \subset \mathcal{K}$.

Remark 10: The condition in (47) determines whether the intersection of two polymatroids is either an active or an inactive case with respect to the $K$-user sum-rate. 

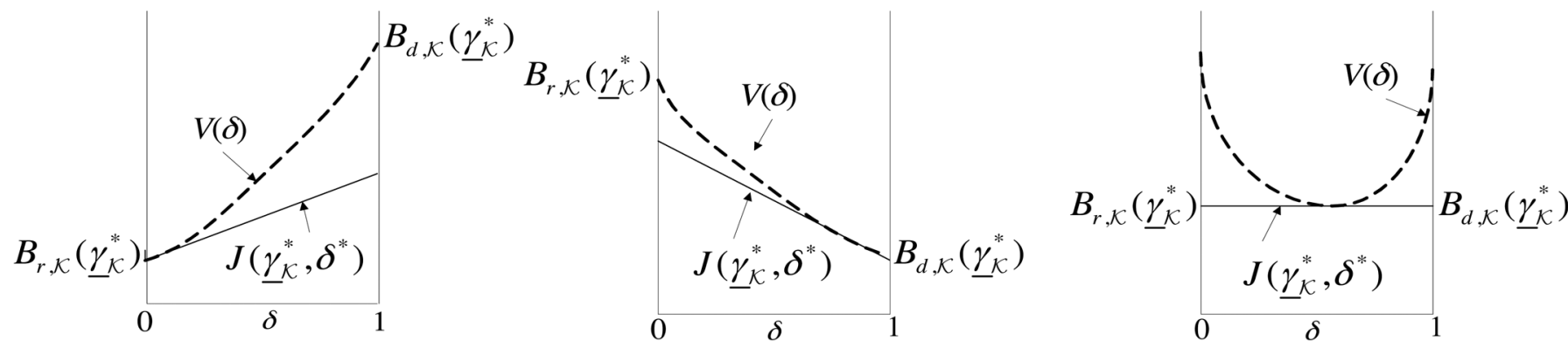

Fig. 3. Illustration of Cases 1, 2, and 3.

Proof: The proof follows from applying Lemma 1 to the maximization $R_{\mathcal{K}}=\sum_{k \in \mathcal{K}} R_{k}$ for each choice of $\left(\underline{\alpha}_{\mathcal{K}}, \underline{\beta}_{\mathcal{K}}\right)$.

From (42), we see that $R_{\mathcal{K}}^{\mathrm{ob}}\left(\underline{\gamma}_{\mathcal{K}}\right)$ does not exceed the minimum of the two $K$-user sum-rate outer bounds $B_{r, \mathcal{K}}\left(\underline{\gamma}_{\mathcal{K}}\right)$ and $B_{d, \mathcal{K}}\left(\underline{\gamma}_{\mathcal{K}}\right)$. Similarly, from (44), $R_{\mathcal{K}}^{\mathrm{DF}}\left(\underline{\alpha}_{\mathcal{K}}, \underline{\beta}_{\mathcal{K}}\right)$ does not exceed the minimum of the two $K$-user sum-rate inner bounds $I_{r, \mathcal{K}}\left(\underline{\alpha}_{\mathcal{K}}\right)$ and $I_{d, \mathcal{K}}\left(\underline{\alpha}_{\mathcal{K}}, \underline{\beta}_{\mathcal{K}}\right)$. Thus, for the inner bounds, we have

$$
\begin{aligned}
R_{\mathcal{K}}^{\mathrm{DF}}\left(\underline{\alpha}_{\mathcal{K}}, \underline{\beta}_{\mathcal{K}}\right) & \leq \min \left(I_{r, \mathcal{K}}\left(\underline{\alpha}_{\mathcal{K}}, \underline{\beta}_{\mathcal{K}}\right), I_{d, \mathcal{K}}\left(\underline{\alpha}_{\mathcal{K}}, \underline{\beta}_{\mathcal{K}}\right)\right) \\
& \leq \max _{\left(\underline{\alpha}_{\mathcal{K}}, \underline{\beta}_{\mathcal{K}}\right)} \min \left(I_{r, \mathcal{K}}\left(\underline{\alpha}_{\mathcal{K}}, \underline{\beta}_{\mathcal{K}}\right), I_{d, \mathcal{K}}\left(\underline{\alpha}_{\mathcal{K}}, \underline{\beta}_{\mathcal{K}}\right)\right) .
\end{aligned}
$$

Similarly, we have

$$
\begin{aligned}
R_{\mathcal{K}}^{\mathrm{ob}}\left(\underline{\gamma}_{\mathcal{K}}\right) & \leq \min \left(B_{r, \mathcal{K}}\left(\underline{\gamma}_{\mathcal{K}}\right), B_{d, \mathcal{K}}\left(\underline{\gamma}_{\mathcal{K}}\right)\right) \\
& \leq \max _{\underline{\gamma}_{\mathcal{K}}} \min \left(B_{r, \mathcal{K}}\left(\underline{\gamma}_{\mathcal{K}}\right), B_{d, \mathcal{K}}\left(\underline{\gamma}_{\mathcal{K}}\right)\right) .
\end{aligned}
$$

We first consider the problem of maximizing the $K$-user DF sum-rate bounds. Based on this analysis, we will argue that it is sufficient to consider the largest $K$-user sum-rate outer bounds in (49).

\section{A. DF: Maximum K-User Sum-Rate}

Consider the optimization problem

$$
R_{\mathcal{K}}=\max _{\left(\underline{\alpha}_{\mathcal{K}}, \underline{\beta}_{\mathcal{K}}\right) \in \Gamma} \min \left(I_{r, \mathcal{K}}\left(\underline{\alpha}_{\mathcal{K}}\right), I_{d, \mathcal{K}}\left(\underline{\alpha}_{\mathcal{K}}, \underline{\beta}_{\mathcal{K}}\right)\right) \text {. }
$$

We write $\left(\underline{\alpha}_{\mathcal{K}}^{*}, \underline{\beta}_{\mathcal{K}}^{*}\right)$ to denote a max-min rule optimizing (50) and write $\mathcal{P}$ to denote the set of all $\left(\underline{\alpha}_{\mathcal{K}}^{*}, \underline{\beta}_{\mathcal{K}}^{*}\right)$ maximizing (50). We use a technique similar to that used in finding the minimax detection rule in a two hypothesis testing problem (see, e.g., $[22$, Ch. II.C]). This allows us to show that a general solution to the max-min optimization in (57) simplifies to three cases [22, Ch. II.C] of which two of them correspond to the cases in which the maximum achieved by one of the two functions is smaller than the other, while the third corresponds to the case in which the maximum results when the two functions are equal (see Fig. 3). For $I_{r, \mathcal{K}}\left(\underline{\alpha}_{\mathcal{K}}\right)$ and $I_{d, \mathcal{K}}\left(\underline{\alpha}_{\mathcal{K}}, \underline{\beta}_{\mathcal{K}}\right)$ defined in (22) and (23), respectively, we can show that the solution simplifies to the consideration of only two cases. The following theorem summarizes the solution to the max-min problem in (50). The detailed proof is developed in Appendix IV.
In the following theorem and the sequel, we write $\underline{1}$ and $\underline{0}$ to denote $K$-length vectors whose entries are all unity and zero, respectively.

Theorem 4: The max-min optimization in (50) simplifies to the following two cases:

Case 1: $\quad R_{\mathcal{K}}=C\left(\frac{\sum_{k \in \mathcal{K}} P_{k}}{N_{r}}\right)$, if $I_{r, \mathcal{K}}(\underline{1})<I_{d, \mathcal{K}}(\underline{1}, \underline{0})$

$$
\text { Case 2: } \quad R_{\mathcal{K}}=I^{*}, \text { if } I_{r, \mathcal{K}}\left(\underline{\alpha}_{\mathcal{K}}^{*}\right)=I_{d, \mathcal{K}}\left(\underline{\alpha}_{\mathcal{K}}^{*}, \underline{\beta}_{\mathcal{K}}^{*}\right)
$$

where $I^{*}$ is given by (32), $P_{\max }$ and $\lambda_{k}$ are given by (33), the entries of the optimal $\beta_{\mathcal{K}}^{*}$ are given by (28), and $q^{*}$, defined in (29), is the unique value satisfying the quadratic $I_{r, \mathcal{K}}\left(\underline{\alpha}_{\mathcal{K}}^{*}\right)=$ $I_{d, \mathcal{K}}\left(\underline{\alpha}_{\mathcal{K}}^{*}, \underline{\beta}_{\mathcal{K}}^{*}\right)$ given by (30).

Proof: Using techniques similar to those used in minimax hypothesis testing, in Appendix IV we show that the following three cases result:

Case 1: $\quad I_{r, \mathcal{K}}(\underline{1})<I_{d, \mathcal{K}}\left(\underline{1}, \underline{\beta}_{\mathcal{K}}\right)=I_{d, \mathcal{K}}(\underline{1}, \underline{0})$

Case 2: $\quad I_{r, \mathcal{K}}\left(\underline{\alpha}_{\mathcal{K}}^{*}\right)=I_{d, \mathcal{K}}\left(\underline{\alpha}_{\mathcal{K}}^{*}, \underline{\beta}_{\mathcal{K}}^{*}\right)$

Case 3: $I_{r, \mathcal{K}}(\underline{0})>I_{d, \mathcal{K}}\left(\underline{0}, \underline{\beta}_{\mathcal{K}}^{*}\right)$.

From (22), we see that $I_{r, \mathcal{K}}\left(\underline{\alpha}_{\mathcal{K}}\right)$ is maximized for $\underline{\alpha}_{\mathcal{K}}=\underline{1}$. In Appendix II, it is shown that $I_{d, \mathcal{K}}\left(\underline{\alpha}_{\mathcal{K}}, \underline{\beta}_{\mathcal{K}}\right)$ is a concave function of $\left(\underline{\alpha}_{\mathcal{K}}, \underline{\beta}_{\mathcal{K}}\right)$ and is maximized for $\underline{\alpha}_{\mathcal{K}}=\underline{0}$ and $\underline{\beta}_{\mathcal{K}}^{*}$ given by (28).

Case 1: From (52), Case 1 results when the maximum $K$-user sum-rate at the relay, $I_{r, \mathcal{K}}(\underline{1})$, is less than the corresponding rate at the destination, $I_{d, \mathcal{K}}\left(\underline{1}, \underline{\beta}_{\mathcal{K}}\right)=I_{d, \mathcal{K}}(\underline{1}, \underline{0})$, where we have used the fact that $I_{d, \mathcal{K}}$ is independent of $\underline{\beta}_{\mathcal{K}}$ for $\underline{\alpha}_{\mathcal{K}}=\underline{1}$. The resulting condition for Case 1 is

$$
\text { Case } 1 \text { Condition: } \frac{\sum_{k \in \mathcal{K}} P_{k}}{N_{r}}<\frac{\sum_{k \in \mathcal{K}} P_{k}+P_{r}}{N_{d}} .
$$

Thus, for this case, each source allocates all its power to transmitting a new message, i.e., does not allocate any power to cooperating with the relay.

Case 3: Since $I_{d, \mathcal{K}}\left(\underline{\alpha}_{\mathcal{K}}, \underline{\beta}_{\mathcal{K}}\right)$ is maximized for $\underline{\alpha}_{\mathcal{K}}=\underline{0}$, Case 3 results when $I_{d, \mathcal{K}}\left(\underline{0}_{\mathcal{K}}, \underline{\beta}_{\mathcal{K}}^{\mathrm{K}}\right)<I_{r, \mathcal{K}}\left(\underline{0}_{\mathcal{K}}\right)$. One can verify that $I_{d, \mathcal{K}}\left(\underline{0}_{\mathcal{K}}, \underline{\beta}_{\mathcal{K}}^{*}\right)>0$. However, from $(22), I_{r, \mathcal{K}}(\underline{0})=0$, and 
thus, since the condition for this case in (52) cannot be satisfied, this case is infeasible.

Case 2: Finally, for Case 2, we first show that since $I_{r, \mathcal{K}}\left(\underline{\alpha}_{\mathcal{K}}\right)$ is independent of $\underline{\beta}_{\mathcal{K}}$, it is sufficient to choose $\underline{\beta}_{\mathcal{K}}^{*}$ in (28) to maximize $I_{d, \mathcal{K}}\left(\underline{\alpha}_{\mathcal{K}}, \underline{\beta}_{\mathcal{K}}\right)$ which in turn simplifies $I_{d, \mathcal{K}}\left(\underline{\alpha}_{\mathcal{K}}, \underline{\beta}_{\mathcal{K}}^{*}\right)$ to be purely a function of $\underline{\alpha}_{\mathcal{K}}$. For $q$ defined in (29), we can further simplify $I_{d, \mathcal{K}}\left(\underline{\alpha}_{\mathcal{K}}, \underline{\beta}_{\mathcal{K}}^{*}\right)$ and $I_{r, \mathcal{K}}\left(\underline{\alpha}_{\mathcal{K}}\right)$ as functions of $q$ and show that they are monotonically increasing and decreasing functions of $q$, respectively. Thus, a unique $q^{*}$ satisfies $I_{d, \mathcal{K}}\left(\underline{\alpha}_{\mathcal{K}}^{*}, \underline{\beta}_{\mathcal{K}}^{*}\right)=I_{r, \mathcal{K}}\left(\underline{\alpha}_{\mathcal{K}}^{*}\right)$ and is given by (30) such that $I_{d, \mathcal{K}}\left(q^{*}\right)=\overline{I_{r}, \mathcal{K}}\left(q^{*}\right)=I^{*}$ where $I^{*}$ is defined in (51). Since (29) describes a $K$-dimensional plane in the $\underline{\alpha}_{\mathcal{K}}$ space, the max-min rule for Case 2 is given by the set

$$
\begin{aligned}
\mathcal{P}=\left\{\left(\underline{\alpha}_{\mathcal{K}}^{*}, \underline{\beta}_{\mathcal{K}}^{*}\right): \underline{\alpha}_{\mathcal{K}}^{*}\right. \text { satisfies (29) and } \\
\left.\underline{\beta}_{\mathcal{K}}^{*} \text { satisfies (28) for every } \underline{\alpha}_{\mathcal{K}}^{*}\right\} .
\end{aligned}
$$

The three cases are mutually exclusive such that Case 2 results when the condition for the other two cases are not satisfied. Since Case 3 is not feasible, the condition for Case 2 is

$$
\text { Case } 2 \text { Condition: } \frac{\sum_{k \in \mathcal{K}} P_{k}}{N_{r}} \geq \frac{\sum_{k \in \mathcal{K}} P_{k}+P_{r}}{N_{d}} \text {. }
$$

The expressions $B_{r, \mathcal{K}}$ and $B_{d, \mathcal{K}}$ for the $K$-user sum-rate outer bounds do not exactly match $I_{r, \mathcal{K}}$ and $I_{d, \mathcal{K}}$, respectively, for the inner bounds. However, we now show that it suffices to consider the largest outer bounds obtained by maximizing the minimum of $B_{r, \mathcal{K}}\left(\underline{\gamma}_{\mathcal{K}}\right)$ and $B_{d, \mathcal{K}}\left(\underline{\gamma}_{\mathcal{K}}\right)$ over all $\underline{\gamma}_{\mathcal{K}} \in \Gamma_{\text {OB }}$. Our motivation to do so stems from the observation that for both the inner and outer bounds, the sum-rate maximization simplifies to the same bounds and thus, it is sufficient to consider only the largest outer bounds.

\section{B. Outer Bounds: Maximum K-User Sum-Rate}

Consider the optimization problem

$$
R_{\mathcal{K}}^{\mathrm{OB}}=\max _{\underline{\gamma}_{\mathcal{K}} \in \Gamma_{\text {ов }}} \min \left\{B_{r, \mathcal{K}}\left(\underline{\gamma}_{\mathcal{K}}\right), B_{d, \mathcal{K}}\left(\underline{\gamma}_{\mathcal{K}}\right)\right\} .
$$

Analogously to the DF analysis, one can similarly show that the max-min problem in (57) simplifies to considering three cases. For $B_{r, \mathcal{K}}$ and $B_{d, \mathcal{K}}$ defined in (16) and (17), respectively, one can show that the solution simplifies to the consideration of only two cases. The following theorem summarizes the solution to the max-min problem in (57). The proof is similar to that for the inner bounds and is omitted in the interest of space.

Theorem 5: The max-min optimization in (57) simplifies to the following two cases:

$$
\begin{gathered}
\text { Case 1: } \quad R_{\mathcal{K}}=C\left(\frac{\sum_{k \in \mathcal{K}} P_{k}}{N_{r}}\right), \text { if } B_{r, \mathcal{K}}(\underline{0})<B_{d, \mathcal{K}}(\underline{0}) \\
\text { Case 2: } \quad R_{\mathcal{K}}=C\left(\left(\sum_{k \in \mathcal{K}} \frac{P_{k}}{N_{r}}\right)-\frac{\left(x^{*}\right)^{2} P_{\max }}{N_{r}}\right)=I^{*}, \\
\quad \text { if } B_{r, \mathcal{K}}^{*}\left(\underline{\gamma}_{\mathcal{K}}^{*}\right)=B_{d, \mathcal{K}}^{*}\left(\underline{\gamma}_{\mathcal{K}}^{*}\right)
\end{gathered}
$$

where $P_{\max }$ and $\lambda_{k}$ are given by (33)

$$
x \triangleq \sum_{k \in \mathcal{K}} \sqrt{\lambda_{k} \gamma_{k}}
$$

such that $x^{*}$ is the unique solution satisfying $B_{r . \mathcal{K}}\left(\underline{\gamma}_{\mathcal{K}}^{*}\right)=$ $B_{r . \mathcal{K}}\left(\underline{\gamma}_{\mathcal{K}}^{*}\right)$ and is given by

$$
x^{*}=q^{*}
$$

for $q^{*}$ given by (30) and (31).

Proof: The proof of Theorem 5 follows along the same lines as that for Theorem 4, and thus, we briefly outline the proof below. Using techniques similar to minimax hypothesis testing, we can show that the following three cases result:

$$
\begin{array}{ll}
\text { Case 1: } & B_{r, \mathcal{K}}(\underline{0})<B_{d, \mathcal{K}}(\underline{0}) \\
\text { Case 2: } & B_{r, \mathcal{K}}\left(\underline{\gamma}_{\mathcal{K}}^{*}\right)=B_{d, \mathcal{K}}\left(\underline{\gamma}_{\mathcal{K}}^{*}\right) \\
\text { Case 3: } & B_{r, \mathcal{K}}\left(\underline{\gamma}_{\mathcal{K}}^{*}\right)>B_{d, \mathcal{K}}\left(\underline{\gamma}_{\mathcal{K}}^{*}\right) .
\end{array}
$$

From (16), we see that $B_{r, \mathcal{K}}\left(\underline{\gamma}_{\mathcal{K}}\right)$ is maximized for $\underline{\gamma} \mathcal{K}=\underline{0}$. In Appendix II, it is shown that $\bar{B}_{d, \mathcal{K}}\left(\underline{\gamma}_{\mathcal{K}}\right)$ is a concave function of $\underline{\gamma}_{\mathcal{K}}$ and is maximized for

$$
\gamma_{k}^{*}=\left\{\frac{P_{k}}{\sum_{k \in \mathcal{K}} P_{k}}, \quad \text { for all } k \in \mathcal{K} .\right.
$$

Case 1: From (61), Case 1 results when the maximum $K$-user sum-rate $B_{r, \mathcal{K}}(\underline{0})$ is less than $B_{d, \mathcal{K}}(\underline{0})$. Since $B_{r, \mathcal{K}}(\underline{0})=I_{r, \mathcal{K}}(\underline{1})$ and $B_{d, \mathcal{K}}(\underline{0})=I_{d, \mathcal{K}}(\underline{1}, \underline{0})$, the condition for this case is the same as that for DF under Case 1, i.e., it is given by (53).

Case 3: Since $B_{d, \mathcal{K}}\left(\underline{\gamma}_{\mathcal{K}}\right)$ is maximized by a $\underline{\gamma}_{\mathcal{K}}^{*}$ with entries given by (62), Case 3 results when $B_{d, \mathcal{K}}\left(\underline{\gamma}_{\mathcal{K}}^{*}\right) \stackrel{-\mathcal{K}}{<} B_{r, \mathcal{K}}\left(\underline{\gamma}_{\mathcal{K}}^{*}\right)$. One can verify that $B_{d, \mathcal{K}}\left(\underline{\gamma}_{\mathcal{K}}^{*}\right)>0$. However, from (16), $B_{r, \mathcal{K}}\left(\underline{\gamma}_{\mathcal{K}}^{*}\right)=0$, and thus, this case is infeasible.

Case 2: Finally, for Case 2, using (59), we can simplify $B_{r, \mathcal{K}}\left(\underline{\gamma}_{\mathcal{K}}\right)$ and $B_{d, \mathcal{K}}\left(\underline{\gamma}_{\mathcal{K}}\right)$ as functions of $x$. As a result, we have $B_{r, \mathcal{K}}(x)=I_{r, \mathcal{K}}(x)$ and $B_{d, \mathcal{K}}(x)=I_{d, \mathcal{K}}(x)$. Thus, we obtain $x^{*}=q^{*}$ such that $B_{r, \mathcal{K}}\left(x^{*}\right)=B_{d, \mathcal{K}}\left(x^{*}\right)=I^{*}$. Since (59) describes a $K$-dimensional plane in the $\underline{\gamma}_{\mathcal{K}}$ space, the $\underline{\gamma}_{\mathcal{K}}^{*}$ for Case 2 is given by the set

$$
\mathcal{G}=\left\{\underline{\gamma}_{\mathcal{K}}^{*}: \underline{\gamma}_{\mathcal{K}}^{*} \text { satisfies (59) }\right\}
$$

Finally, since the three cases are mutually exclusive, and Case 3 is not feasible, the condition for Case 2 is the same as that for DF in (56).

Thus, from Theorems 4 and 5 we see that the maximization in (50) and (57) yields the same solution. Furthermore, the maximizations in (50) and (57) are independent of whether the max-min rules $\left(\underline{\alpha}_{\mathcal{K}}^{*}, \underline{\beta}_{\mathcal{K}}^{*}\right)$ and $\underline{\gamma}_{\mathcal{K}}^{*}$, respectively, results in an active or an inactive case.

In the following section, we show that for the condition for Case 1 implies an active case. For Case 2, not all max-min rules will result in an active case. However, irrespective of the kind of intersection, we have that the largest inner and outer bounds on the $K$-user sum-rate are equal. 


\section{CAPACITY THEOREMS}

In this section, we develop a proof for Theorem 3. The following theorems help us in developing a proof for Theorem 3.

Theorem 6: For a degraded Gaussian MARC in which the source and relay powers satisfy the Case 1 condition

$$
\frac{\sum_{k \in \mathcal{K}} P_{k}}{N_{r}}<\frac{\left(\sum_{k \in \mathcal{K}} P_{k}\right)+P_{r}}{N_{d}},
$$

the $K$-user sum-capacity $C_{\mathcal{K}}$ and capacity region $\mathcal{C}_{\text {MARC }}$ are given by

$$
\begin{aligned}
& C_{\mathcal{K}}=C\left(\sum_{k \in \mathcal{K}} \frac{P_{k}}{N_{r}}\right), \text { and } \\
& \mathcal{C}_{\text {MARC }}=\mathcal{R}_{r}(\underline{1})
\end{aligned}
$$

where $\mathcal{R}_{r}(\underline{1})$ is given by (24).

Proof: The proof of (64) follows directly from comparing the largest inner and outer bounds on $R_{\mathcal{K}}$ for Case 1 in Theorems 4 and 5 . We now prove that DF also achieves the capacity region for Case 1 .

Let $\underline{\alpha}_{\mathcal{K}}=\underline{1}$ and $\underline{\beta}_{\mathcal{K}}=\underline{0}$. For this choice of $\left(\underline{\alpha}_{\mathcal{K}}, \underline{\beta}_{\mathcal{K}}\right)$, the bounds $I_{r, \mathcal{S}}$ and $I_{d, \mathcal{S}}$ can be expanded for this case using (22) and (23), respectively, as

$$
\begin{aligned}
I_{r, \mathcal{S}}(\underline{1}) & =C\left(\frac{\sum_{k \in \mathcal{S}} P_{k}}{N_{r}}\right) \\
I_{d, \mathcal{S}}(\underline{1}, \underline{0}) & =C\left(\frac{\sum_{k \in \mathcal{S}} P_{k}}{N_{d}}+\frac{P_{r}}{N_{d}}\right) .
\end{aligned}
$$

The condition for Case 1 in Theorem 4 requires

$$
\frac{\sum_{k \in \mathcal{K}} P_{k}}{N_{r}} \leq \frac{\sum_{k \in \mathcal{K}} P_{k}}{N_{d}}+\frac{P_{r}}{N_{d}} .
$$

Expanding (68), we have, for any $\mathcal{S} \subset \mathcal{K}$

$$
\begin{aligned}
\frac{\sum_{k \in \mathcal{S}} P_{k}}{N_{r}} & \leq \frac{\sum_{k \in \mathcal{S}} P_{k}+P_{r}}{N_{d}}-\frac{\sum_{k \in \mathcal{S}^{c}} P_{k}\left(N_{d}-N_{r}\right)}{N_{d} N_{r}} \\
& <\frac{\sum_{k \in \mathcal{S}} P_{k}+P_{r}}{N_{d}}
\end{aligned}
$$

where (70) follows from (4). Thus, $I_{r, \mathcal{K}}(\underline{1}) \leq I_{d, \mathcal{K}}(\underline{1}, \underline{0})$ implies that $I_{r, \mathcal{S}}(\underline{1})<I_{d, \mathcal{S}}(\underline{1}, \underline{0})$ for all $\mathcal{S} \subset \mathcal{K}$, i.e., $\mathcal{R}_{r}(\underline{1}) \subset \mathcal{R}_{d}(\underline{1}, \underline{0})$. Recall that we chose $\underline{\beta}_{\mathcal{K}}=\underline{0}$. From (22), we see that the choice of $\underline{\beta}_{\mathcal{K}}$ does not affect $\mathcal{R}_{r}$. Further, a nonzero $\underline{\beta}_{\mathcal{K}}$ does not increase $I_{d, \mathcal{K}}$. However, it can decrease $I_{d, \mathcal{S}}$ for some or all $\mathcal{S} \subset \mathcal{K}$ as

$$
\begin{aligned}
I_{d, \mathcal{S}}\left(\underline{1}, \underline{\beta}_{\mathcal{K}}\right) & =C\left(\frac{\left(\sum_{k \in \mathcal{S}} P_{k}\right)+P_{r}\left(1-\sum_{k \in \mathcal{S}^{c}} \beta_{k}\right)}{N_{d}}\right) \\
& \leq I_{d, \mathcal{S}}(\underline{1}, \underline{0})
\end{aligned}
$$

i.e.,

$$
\mathcal{R}_{r}(\underline{1}) \cap \mathcal{R}_{d}\left(\underline{1}, \underline{\beta}_{\mathcal{K}} \neq \underline{0}\right) \subset \mathcal{R}_{r}(\underline{1}) \cap \mathcal{R}_{d}(\underline{1}, \underline{0})=\mathcal{R}_{r}(\underline{1}) .
$$

Finally, since for Case $1, I_{r, \mathcal{S}}(\underline{1})=B_{r, \mathcal{S}}(\underline{0})$ and $I_{d, \mathcal{S}}(\underline{1}, \underline{0})=$ $B_{d, \mathcal{S}}(\underline{0}), \mathrm{DF}$ achieves the capacity region for Case 1 since

$$
\mathcal{R}_{r}(\underline{1})=\mathcal{R}_{r}^{\mathrm{ob}}(\underline{0}) \subset \mathcal{R}_{d}(\underline{1}, \underline{0})=\mathcal{R}_{d}^{\mathrm{ob}}(\underline{0}) .
$$

This proves (65). Note that the optimal signaling scheme for Case 1 is for the sources to use all their power to transmit a new message.

We now consider Case 2. The following theorem summarizes our results for this case.

Theorem 7: For a degraded Gaussian MARC in which the source and relay powers satisfy the Case 2 condition

$$
\frac{\sum_{k \in \mathcal{K}} P_{k}}{N_{r}} \geq \frac{\left(\sum_{k \in \mathcal{K}} P_{k}\right)+P_{r}}{N_{d}}
$$

the $K$-user sum-capacity $C_{\mathcal{K}}$ is

$$
\begin{array}{ll}
C_{\mathcal{K}}=I^{*} & \text { if there exists an }\left(\underline{\alpha}_{\mathcal{K}}^{*}, \underline{\beta}_{\mathcal{K}}^{*}\right) \in \mathcal{P} \text { s.t. } \\
& \mathcal{R}_{r}\left(\underline{\alpha}_{\mathcal{K}}^{*}\right) \cap \mathcal{R}_{d}\left(\underline{\alpha}_{\mathcal{K}}^{*}, \underline{\beta}_{\mathcal{K}}^{*}\right) \text { is an active case. } \\
C_{\mathcal{K}}<I^{*} & \text { if } \mathcal{R}_{r}\left(\underline{\alpha}_{\mathcal{K}}^{*}\right) \cap \mathcal{R}_{d}\left(\underline{\alpha}_{\mathcal{K}}^{*}, \underline{\beta}_{\mathcal{K}}^{*}\right) \text { is an } \\
& \text { inactive case for all }\left(\underline{\alpha}_{\mathcal{K}}^{*}, \underline{\beta}_{\mathcal{K}}^{*}\right) .
\end{array}
$$

For the class of symmetric degraded Gaussian MARCs

$$
C_{\mathcal{K}}=I^{*}, \quad \text { for } \alpha_{k}^{*}=\alpha^{*}=\left(q^{*}\right)^{2} / K .
$$

Proof: From Theorems 4 and 5, when the condition in (71) is satisfied, the largest DF bound on the $K$-user sum-rate matches the largest $K$-user sum-rate outer bound. In fact, this bound is achievable only if there exists at least one max-min rule $\left(\underline{\alpha}_{\mathcal{K}}^{*}, \underline{\beta}_{\mathcal{K}}^{*}\right) \in \mathcal{P}$ for which $\mathcal{R}_{r}\left(\underline{\alpha}_{\mathcal{K}}^{*}\right) \cap \mathcal{R}_{d}\left(\underline{\alpha}_{\mathcal{K}}^{*}, \underline{\beta}_{\mathcal{K}}^{*}\right)$ is an active case.

Let $\mathcal{P}_{a} \subseteq \mathcal{P}$ denote the set of $\left(\underline{\alpha}_{\mathcal{K}}^{*}, \underline{\beta}_{\mathcal{K}}^{*}\right)$ that result in active cases. From Lemma 6 and Theorem 4, we can write the maximum $K$-user DF sum-rate when $I_{r, \mathcal{K}}(\underline{1})>I_{d, \mathcal{K}}(\underline{1}, \underline{0})$ as

$$
R_{\mathcal{K}}= \begin{cases}S_{a}\left(\underline{\alpha}_{\mathcal{K}}^{*}, \underline{\beta}_{\mathcal{K}}^{*}\right)=I^{*}, & \text { if }\left(\underline{\alpha}_{\mathcal{K}}^{*}, \underline{\beta}_{\mathcal{K}}^{*}\right) \in \mathcal{P}_{a} \\ \max _{\mathcal{P}, \mathcal{A} \subset \mathcal{K}} S_{i}\left(\underline{\alpha}_{\mathcal{K}}^{*}, \underline{\beta}_{\mathcal{K}}^{*}\right)<I^{*}, & \text { if } \mathcal{P}_{a}=\emptyset\end{cases}
$$

where $S_{a}(\cdot)$ and $S_{i}(\cdot)$ are given by (46) and (45), respectively, and $I^{*}$ is given by (32).

In Theorem 4, it was shown that when (71) holds, the $\max$-min rules $\left(\underline{\alpha}_{\mathcal{K}}^{*}, \underline{\beta}_{\mathcal{K}}^{*}\right)$ are such that $\underline{\alpha}_{\mathcal{K}}^{*} \neq \underline{1}$ and $\underline{\beta}_{\mathcal{K}}^{*} \neq \underline{0}$. However, for any max-min rule $\left(\underline{\alpha}_{\mathcal{K}}^{*}, \underline{\beta}_{\mathcal{K}}^{*}\right)$ it is not straightforward to show that an active case results.

We now show that for class of symmetric Gaussian MARC channels, when the condition in (71) holds, DF achieves the $K$-user sum-capacity. For this class, since $P_{k}=P$, from symmetry, $I_{d, \mathcal{K}}=I_{r, \mathcal{K}}$ in (23) can be maximized by choosing $\alpha_{k}^{*}=\alpha^{*}$ for all $k$ in (29) such that

$$
\left(1-\alpha^{*}\right)=\left(q^{*}\right)^{2} / K \text {. }
$$

From (29), since $0<\left(q^{*}\right)^{2}<\sum_{k=1}^{K} \lambda_{k}=K$, there exists an $0<\alpha^{*}<1$ that achieves $I^{*}$ in (72).

From symmetry, no subset of users achieves a larger rate at one of the receiver than any other subset, i.e., for $\alpha_{k}^{*}=\alpha^{*}$ and $\beta_{k}=1 / K$, for all $k, \mathcal{R}_{r}\left(\underline{\alpha}_{\mathcal{K}}^{*}\right) \cap \mathcal{R}_{d}\left(\underline{\alpha}_{\mathcal{K}}^{*}, \underline{\beta}_{\mathcal{K}}^{*}\right)$ is an active case. Thus, the maximum sum-rate $I^{*}$ is achievable, and since, this is the largest outer bound on the $K$-user sum-rate, $\mathrm{DF}$ achieves the sum-capacity for the symmetric class of channels. 
For completeness, one can also show that for the outer bounds when (71) is satisfied, a $\underline{\gamma}_{\mathcal{K}}^{*}$ whose entries $\gamma_{k}^{*}=\gamma^{*}$, for all $k$, results in an active case. The proof follows simply from setting

$$
\gamma_{k}=\left(1-\alpha_{k}\right) \beta_{k}, \quad \text { where }\left(\underline{\alpha}_{\mathcal{K}}, \underline{\beta}_{\mathcal{K}}\right) \in \Gamma .
$$

That (74) satisfies (12) follows from the fact that since $\beta_{k} \leq 1$ for all $k$, we have

$$
\sum_{k \in \mathcal{K}} \gamma_{k}=\sum_{k \in \mathcal{K}}\left(1-\alpha_{k}\right) \beta_{k}<1 .
$$

For the symmetric case, this implies that there exists a

$$
\gamma^{*}=\left(1-\alpha^{*}\right) / K
$$

satisfying (75). In fact, for $\alpha^{*}$ in (73), we obtain $\gamma^{*}=$ $\left(q^{*}\right)^{2} / K^{2}=\left(x^{*}\right)^{2} / K^{2}<1$, i.e., the symmetric $\gamma^{*}$ in (76) is feasible and results in an active case.

Combining the above two theorems, we obtain the proof for Theorem 3. We now present arguments supporting Conjecture 1 in Section III.

\section{A. Arguments for Conjecture 1}

From Theorems 6 and 7, we see that the class of degraded Gaussian MARCs can be divided into two disjoint subclasses. Furthermore, in Theorems 6 and 7, we have proved Conjecture 1 for one of the subclasses and for a special case of the other subclass.

The first subclass is one in which the source and relay SNRs satisify a condition identified as Case 1 throughout the sequel. The condition requires the SNR on the relay to destination link to be sufficiently large that the bottleneck link on the $K$-user sum-rate is the multiple-access link from the sources to the relay. For this subclass, we have shown that the entire region $\mathcal{R}_{r}(\underline{1})$ achieved at the relay lies within that achieved at the destination, and that DF achieves the capacity region of a degraded Gaussian MARC for this subclass.

The second subclass is the class of degraded Gaussian MARCs for which the condition of Case 1 does not hold. For this subclass, using Theorems 4 and 5, we show that the largest bound on the $K$-user sum-rate results when the sum-rate to the relay from the sources is reduced from the maximum $C\left(\sum_{k \in \mathcal{K}} P_{k} / N_{r}\right)$ by reducing some or all $\alpha_{k}$, thereby increasing the sum-rate at the destination, until the $K$-user sum-rates at both receivers are the same. The set of max-min rules, i.e., $\left(\underline{\alpha}_{\mathcal{K}}^{*}, \underline{\beta}_{\mathcal{K}}^{*}\right)$, that achieve the equal sum-rate requirement are given by (28)-(30).

For this subclass, using Theorems 4 and 5, in Theorem 7 we prove that DF achieves the sum-capacity if there exists a max-min rule $\left(\underline{\alpha}_{\mathcal{K}}^{*}, \underline{\beta}_{\mathcal{K}}^{*}\right)$ such that $\mathcal{R}_{r}\left(\underline{\alpha}_{\mathcal{K}}^{*}\right) \cap \mathcal{R}_{d}\left(\underline{\alpha}_{\mathcal{K}}^{*}, \underline{\beta}_{\mathcal{K}}^{*}\right)$ is an active case. Specifically, for the class of symmetric degraded Gaussian MARCs, we showed that $\alpha_{k}^{*}=\alpha=\left(q_{K}^{*}\right)^{2} / K<1$ results in an active case and achieves the sum-capacity.

Since we can show the optimality of DF for the first disjoint subclass and under symmetric source powers for the second subclass, Conjecture 1 addresses the second subclass. In fact, it implies that an active case will always occur for the second subclass, irrespective of source powers.
However, for the second subclass with arbitrary source powers, showing that at least one max-min rule results in an active intersection does not appear straightforward. Here we argue that it will be so, thereby supporting Conjecture 1 .

Our intuition for Conjecture 1 stems from the fact that the physically degraded condition implies that if the sources transmit at rates that allow reliable decoding at the destination, then their signals can also be decoded by the relay. Thus, in general, for the second subclass, there must be an active case. Clearly, for the symmetric case this results from setting $\alpha_{k}^{*}=\alpha^{*}=\left(q^{*}\right)^{2} / K$ for all $k$.

Consider now the asymmetric case where the sources powers are not all the same. Without loss of generality, consider $P_{1}>$ $P_{2}>\cdots>P_{K}$ such that $P_{\max }=P_{1}$. In Theorem 4, we showed that the solution $q^{*}$ satisfying (30) is given by

$$
q^{*}=\frac{-K_{1}+\sqrt{K_{1}^{2}+\left(K_{3}-K_{2}\right) K_{0}}}{K_{0}}
$$

where

$$
\begin{aligned}
& K_{0}=P_{\max } / N_{r}, K_{1}=\sqrt{P_{\max } P_{r}} / N_{d}, \\
& K_{2}=\frac{\sum_{k \in \mathcal{K}} P_{k}}{N_{d}}+\frac{P_{r}}{N_{d}}, \text { and } K_{3}=\frac{\sum_{k \in \mathcal{K}} P_{k}}{N_{r}}
\end{aligned}
$$

and

$$
\sum_{k \in \mathcal{K}}\left(1-\alpha_{k}^{*}\right) \lambda_{k}=\left(q^{*}\right)^{2}
$$

From (77) and (78), one can see that since only the term $K_{3}-$ $K_{2}$ depends explicitly on the powers of all sources, $\left(q^{*}\right)^{2}$, and hence, $\sum_{k \in \mathcal{K}}\left(1-\alpha_{k}^{*}\right) \lambda_{k}$ scales linearly with $P_{1}, P_{2}, \ldots, P_{K}$ as $\sum_{k \in \mathcal{K}} P_{k}=P_{\max } \sum_{k \in \mathcal{K}} \lambda_{k}$.

Let $q_{\text {sym }}^{*}$ denote the optimal $q^{*}$ for the symmetric class. For the symmetric case where $P_{k}=P_{\max }$, for all $k$, we have $\alpha_{k}^{*}=$ $\left(q_{\text {sym }}^{*}\right)^{2} / K$ for all $k$, where we write $q_{\text {sym }}^{*}$ to denote the symmetric case. When $P_{2}, P_{3}, \ldots, P_{K}$ are reduced from $P_{\max }$, from (77) and (78), we have that $q^{*}$ also decreases from $q_{\mathrm{sym}}^{*}$, and thus, $\left(q^{*}\right)^{2}<\left(q_{\text {sym }}^{*}\right)^{2}<K$, and hence, is a feasible solution.

To argue that DF achieves the sum-capacity, we need to argue that an active intersection exists for some $\underline{\alpha}_{\mathcal{K}}^{*}$ satisfying (79). We conjecture that choosing $\alpha_{k}^{*}=\alpha^{*}=\left(q^{*}\right)^{2} / \sum_{k \in \mathcal{K}} \lambda_{k}$, for all $k$, should suffice. This conjecture is motivated by the observation that for asymmetric source powers, the sources with larger powers achieve larger rates at both the relay and the destination, and vice versa. This is in contrast to an inactive case in which a subset of sources achieve large enough rates at one of the receivers while the remaining subset achieve sufficiently large rates at the other receiver such that the intersection of the two multiple-access polymatroids is an inactive case. Thus, with asymmetric source powers, an inactive case will require an $\underline{\alpha}_{\mathcal{K}}^{*}$ whose entries are not all equal. For example, one could choose $\alpha_{1}=1$ such that source 1 achieves a large rate at the relay and sufficiently smaller values for $\alpha_{k}, k \neq 1$, such that the remaining sources achieve a larger rate at the destination.

Finally, in Appendix V, we show that for the same choice of the $K$ source-relay correlation coefficients for both the inner and outer bounds, the outer cutset bounds are at least as large as 
the inner DF bounds for all $\mathcal{S} \subseteq \mathcal{K}$. This implies that for every $\left(\underline{\alpha}_{\mathcal{K}}^{*}, \underline{\beta}_{\mathcal{K}}^{*}\right) \in \mathcal{P}_{a}$, there exists a $\underline{\gamma}_{\mathcal{K}}^{*}$ with entries

$$
\gamma_{k}^{*}=\left(1-\alpha_{k}^{*}\right) \beta_{k}^{*}, \quad \text { for all } k
$$

that results in an active case for the outer bounds, i.e., DF achieves the sum-capacity for the active class.

\section{B. Numerical Examples}

It is straightforward to find numerical examples for Case 1 where DF achieves the capacity region. We focus on Case 2 and present two examples where DF achieves the sum-capacity of a two-user degraded Gaussian MARCs, with $\mathcal{P}_{a}=\mathcal{P}$ for one and $\mathcal{P}_{a} \subset \mathcal{P}$ for the other.

Example 1: Consider a two-user degraded Gaussian MARC with $P_{1} / N_{r}=6, P_{2} / N_{r}=4, P_{1} / N_{d}=3, P_{2} / N_{d}=2$, and $P_{r} / N_{d}=2$. These SNR values satisfy the condition for Case 2 in (71), and thus, the DF sum-rate is maximized by a set of $\left(\underline{\alpha}_{\mathcal{K}}^{*}, \underline{\beta}_{\mathcal{K}}^{*}\right)$ where $\underline{\alpha}_{\mathcal{K}}^{*}$ satisfies

$$
\left(1-\alpha_{1}^{*}\right)+\frac{2}{3}\left(1-\alpha_{2}^{*}\right)=\left(q^{*}\right)^{2}=0.408
$$

and for every choice of $\underline{\alpha}_{\mathcal{K}}^{*}$ satisfying (80), $\underline{\beta}_{\mathcal{K}}^{*}$ is given by (28). The set of feasible $\underline{\alpha}_{\mathcal{K}}^{*}$ has entries $\alpha_{1}^{*} \in(\overline{0.83}, 1]$ with $\alpha_{2}^{*}$ for each such $\alpha_{1}^{*}$ satisfying (80) such that $\alpha_{2}^{*} \in(0.75,1]$. For these SNR parameters, the set $\mathcal{P}_{a}=\mathcal{P}$ and for each $\left(\underline{\alpha}_{\mathcal{K}}^{*}, \underline{\beta}_{\mathcal{K}}^{*}\right) \in \mathcal{P}$, the correlation values $\gamma_{k}^{*}=\left(1-\alpha_{k}^{*}\right) \beta_{k}^{*}$, for all $k=1,2$. result in the vector $\underline{\gamma}_{\mathcal{K}}^{*} \in \mathcal{G}_{a}$.

Example 2: We next consider a two-user example with $P_{1} / N_{r}=6, P_{2} / N_{r}=0.4, P_{1} / N_{d}=3, P_{2} / N_{d}=0.2$, and $P_{r} / N_{d}=2$. These SNR values also satisfy the condition for Case 2 in (71), and thus, the DF sum-rate is maximized by a set of $\left(\underline{\alpha}_{\mathcal{K}}^{*}, \underline{\beta}_{\mathcal{K}}^{*}\right)$ where $\underline{\alpha}_{\mathcal{K}}^{*}$ satisfies

$$
\left(1-\alpha_{1}^{*}\right)+\frac{1}{15}\left(1-\alpha_{2}^{*}\right)=\left(q^{*}\right)^{2}=0.0389 .
$$

The set of feasible $\underline{\alpha}_{\mathcal{K}}^{*}$ has entries $\alpha_{1}^{*} \in(0.96,1]$ with $\alpha_{2}^{*}$ for each such $\alpha_{1}^{*}$ satisfying (81) such that $\alpha_{2}^{*} \in(0.416,1]$. Note that subject to (81), $\alpha_{2}$ decreases as $\alpha_{1}$ increases and vice versa. For these SNR parameters, the set $\mathcal{P}_{a}$ consists of $\left(\underline{\alpha}_{\mathcal{K}}^{*}, \underline{\beta}_{\mathcal{K}}^{*}\right)$ with entries $\alpha_{1}^{*}$ and $\alpha_{2}^{*}$ that are restricted to $(0.961,0.979]$ and $(0.731,1]$, respectively. Thus, the fractions $\alpha_{1}^{*}=\alpha_{2}^{*}=\alpha^{*}=$ 0.963 , as conjectured, result in an active case.

Finally, for the two-user degraded Gaussian MARC, a numerical example illustrating $\mathcal{P}_{a}=\emptyset$, i.e., the set of max-min rules that result in an active case, does not appear straightforward despite using a wide range of ratios of $P_{1}$ to $P_{2}$, i.e., not all rate-maximizing intersections are such that one of the sources achieve better rates at one of the receivers while the other source achieves a better rate at the other receiver. This is in line with our conjecture, as at any receiver, the noise seen by both sources is the same, and thus, the source with smaller power typically achieves smaller rates at both receivers. It may be possible to increase the rate achieved at the destination by increasing the relay power; however, large values of relay power will result in the bottleneck case for which the condition for Case 1 will hold.
Remark 11: In the above analysis, we determined the sumcapacity for a degraded Gaussian MARC under a per-symbol transmit power constraint at the sources and relay. One can also consider an average power constraint at every transmitter. The achievable strategy remains unchanged; for the converse, we start with the convex sums of the outer bounds in (34) over $n$ channel uses. Recall that $B_{d, \mathcal{S}}$ is a concave function of the correlation coefficients and power. On the other hand, $B_{r, \mathcal{S}}$ for all $\mathcal{S} \subset \mathcal{K}$ is not a concave function of the power and cross-correlation parameters. However, we can use the concavity of $B_{r, \mathcal{K}}$ to show that the maximum bounds on the sum-rate in Theorem 5 remain unchanged. Finally, we note that as with the symbol power constraint, here too we require time sharing to develop the outer bound rate region.

\section{CONCLUDING REMARKS}

In this paper, we have studied the sum-capacity of degraded Gaussian MARCs. In particular, we have developed the rate regions for the achievable strategy of DF and the cutset outer bounds. The outer bounds have been obtained using cut-set bounds for the case of independent sources and have been shown to be maximized by Gaussian signaling at the sources and relay.

We have also shown that, in general, the rate regions achieved by the inner and outer bounds are not the same. This difference is due to the fact that the input distributions and the rate expressions for the inner and outer bounds are not exactly the same. In fact, the input distribution for the inner bound uses auxiliary random variables to model the correlation between the inputs at the sources and the relay and is more restrictive than the distribution for the outer bound. Despite these differences, Gaussian signaling maximizes the outer bounds and can be chosen for the inner bounds. Thus, in both cases the input distributions can be quantified by a set of $K$ source-relay cross-correlation coefficients.

In both cases, we have also shown that the rate region for every choice of the appropriate input distribution is an intersection of polymatroids. We have used the properties of polymatroid intersections to show that the largest inner and outer bounds on the $K$-user sum-rate is at most the maximum of the minimum of the two $K$-user sum-rate bounds, with equality only when the polymatroid intersections is an active case for which the $K$-user sum-rate constraints are active.

Our analysis led us to broadly classify $K$-user degraded Gaussian MARCs into two disjoint subclasses. The first subclass is one where the multiple-access link from the sources to the relay is the bottleneck link for which we have shown that DF achieves the sum-capacity as well as the entire capacity region.

The second subclass is one in which the multiple-access links to both the relay (from the sources) and the destination (from the sources and the relay) are comparable. For this subclass, the largest $K$-user DF sum-rate bound we developed is achievable only if the intersection of the two multiple-access polymatroids is an active case in which the $K$-user sum-rate constraints are active. We have shown that an active case occurs when the sources transmit with the same power (symmetric degraded Gaussian 
MARC) and have presented an argument for our conjecture that DF is sum-capacity optimal for all degraded Gaussian MARCs.

\section{APPENDIX I}

\section{OUTER BOUNDS: PROOF}

We now develop the bounds $B_{r, \mathcal{S}}\left(\underline{\gamma}_{\mathcal{K}}\right)$ and $B_{d, \mathcal{S}}\left(\underline{\gamma}_{\mathcal{K}}\right)$. Recall that we write $B_{r, \mathcal{S}}$ and $B_{d, \mathcal{S}}$ to denote, respectively, the first and second bound on $R_{\mathcal{S}}$ in (36) for a constant $U$. Expanding the bounds on $R_{\mathcal{S}}$ in (36) for a constant $U$, we have

$$
R_{\mathcal{S}} \leq \min \left\{h\left(Y_{r} \mid X_{r} X_{\mathcal{S}^{c}}\right)-h\left(Z_{r}\right), h\left(Y_{d} \mid X_{\mathcal{S}^{c}}\right)-h\left(Z_{d}\right)\right\} .
$$

For a fixed covariance matrix of the input random variables $X_{\mathcal{K}}$ and $X_{r}$, one can apply a conditional entropy maximization theorem [23, Lemma 1] to show that $h\left(Y_{r} \mid X_{r} X_{\mathcal{S}^{c}}\right)$ and $h\left(Y_{d} \mid X_{\mathcal{S}^{c}}\right)$ are maximized by choosing the distribution in (35) as jointly Gaussian. Consider the bound $B_{r, \mathcal{S}}$. Expanding $Y_{r}$, we have

$$
R_{\mathcal{S}} \leq C\left(\frac{E\left[\operatorname{var}\left(\sum_{k \in \mathcal{S}} X_{k} \mid X_{r} X_{\mathcal{S}^{c}}\right)\right]}{N_{r}}\right) .
$$

For Gaussian signals, using the chain rule, we have

$$
E\left[\operatorname{var}\left(\sum_{k \in \mathcal{S}} X_{k} \mid X_{r} X_{\mathcal{S}^{c}}\right)\right]=\frac{\operatorname{det}\left(K_{\underline{A} \mid \underline{C}}\right)}{\operatorname{det}\left(K_{\underline{B} \mid \underline{C}}\right)}
$$

where

$$
\begin{aligned}
\underline{A} & =\left[\begin{array}{ll}
\sum_{k \in \mathcal{S}} X_{k} & X_{r}
\end{array}\right]^{T} \\
\underline{B} & =\left[X_{r}\right] \\
\underline{C} & =\left[X_{\mathcal{S}^{c}}\right]
\end{aligned}
$$

and for random vectors $\underline{X}$ and $\underline{Y}$, the conditional covariance $K_{\underline{X} \mid \underline{Y}}$ is

$$
K_{\underline{X} \mid \underline{Y}}=E\left[(\underline{X}-E[\underline{X} \mid \underline{Y}])(\underline{X}-E[\underline{X} \mid \underline{Y}])^{T}\right]
$$

where $\underline{X}^{T}$ is the transpose of $\underline{X}$. We use the fact that $X_{\mathcal{S}}$ and $X_{\mathcal{S}^{c}}$ are independent to expand (83) as

$$
\begin{aligned}
& E\left[\operatorname{var}\left(\sum_{k \in \mathcal{S}} X_{k} \mid X_{r} X_{\mathcal{S}^{c}}\right)\right]= \\
& \operatorname{var}\left(\sum_{k \in \mathcal{S}} X_{k}\right)-\frac{E^{2}\left[\sum_{k \in \mathcal{S}} X_{k} \tilde{X}_{r, \mathcal{S}}\right]}{P_{r, \mathcal{S}}}
\end{aligned}
$$

where $\tilde{X}_{r, \mathcal{S}} \triangleq\left(X_{r}-E\left[X_{r} \mid X_{\mathcal{S}^{c}}\right]\right)$ is a Gaussian random variable with variance

$$
P_{r, \mathcal{S}}=E\left[\tilde{X}_{r, \mathcal{S}}^{2}\right]=E\left[\operatorname{var}\left(X_{r} \mid X_{\mathcal{S}^{c}}\right)\right] .
$$

Substituting (85) in (82) and using (5) to bound $\operatorname{var}\left(X_{k}\right)$ for all $k$, we obtain

$$
R_{\mathcal{S}} \leq C\left(\frac{\left(\sum_{k \in \mathcal{S}} P_{k}\right)-\frac{1}{P_{r, \mathcal{S}}} E^{2}\left[\sum_{k \in \mathcal{S}} X_{k} \tilde{X}_{r, \mathcal{S}}\right]}{N_{r}}\right)
$$

We define $\gamma_{k}$, for all $k \in \mathcal{K}$, by

$$
E\left[X_{k} X_{r}\right] \triangleq \sqrt{\gamma_{k} P_{k} P_{r}}, \quad 0 \leq \sum_{k=1}^{K} \gamma_{k} \leq 1 .
$$

Using the independence of $X_{k}$ for all $k$ and (88), we write

$$
E\left[\sum_{k \in \mathcal{S}} X_{k} \tilde{X}_{r}\right]=\sum_{k \in \mathcal{S}} E\left[X_{k} X_{r}\right]=\sum_{k \in \mathcal{S}} \sqrt{\gamma_{k} P_{k} P_{r}} .
$$

Next we use (88) to evaluate $P_{r, \mathcal{S}}$. We start by considering the random variable

$$
\hat{X}_{r}=X_{r}-E\left[X_{r} \mid X_{K}\right] .
$$

Using (88) and the independence of $X_{k}$ for all $k$, we can write the variance of $\hat{X}_{r}$ as

$$
E\left[\hat{X}_{r}^{2}\right]=E\left[\operatorname{var}\left(X_{r} \mid X_{K}\right)\right]=\left(1-\gamma_{K}\right) P_{r}
$$

where we used (84) to simplify (90). Continuing thus, we consider the random variable $\bar{X}_{r}=\hat{X}_{r}-E\left[\hat{X}_{r} \mid X_{K-1}\right]$. Using the independence of $X_{k}$ for all $k$, we thus have

$$
\begin{aligned}
E\left[\bar{X}_{r}^{2}\right] & =E\left[\hat{X}_{r}^{2}\right]-E\left[E^{2}\left[\hat{X}_{r} \mid X_{K-1}\right]\right] \\
& =E\left[\operatorname{var}\left(X_{r} \mid X_{K-1} X_{K}\right)\right] \\
& =\left(1-\gamma_{K-1}-\gamma_{K}\right) P_{r} .
\end{aligned}
$$

Generalizing the above, we have

$E\left[\operatorname{var}\left(X_{r} \mid X_{\mathcal{S}^{c}}\right)\right]=\left(1-\sum_{k \in \mathcal{S}^{c}} \gamma_{k}\right) P_{r} \triangleq \bar{\gamma}_{\mathcal{S}^{c}} P_{r}$,

for all $\mathcal{S} \subseteq \mathcal{K}$.

Finally, we substitute (91) and (89) in (87) to simplify the first bound as

$$
R_{\mathcal{S}} \leq \begin{cases}C\left(\sum_{k \in \mathcal{S}} \frac{P_{k}}{N_{r}}\right), & \text { if } \sum_{k \in \mathcal{S}^{c}} \gamma_{k}=1 \\ C\left(\sum_{k \in \mathcal{S}} \frac{P_{k}}{N_{r}}-\frac{\left.\left(\sum_{k \in \mathcal{S}} \sqrt{\gamma_{k} P_{k}}\right)^{2}\right),}{N_{r} \bar{\gamma}_{\mathcal{S}}}\right) & \text { otherwise. }\end{cases}
$$

Observe that for $K=1$, we have $V_{1}=X_{r}$ and $\gamma_{1}=1$, and thus, (16) simplifies to the first outer bound in [3, Theorem 5] for the classic single source degraded relay channel. Finally, from (91), observe that $\gamma_{k}$, for all $k$, satisfies

$$
\sum_{k \in \mathcal{K}} \gamma_{k} \leq 1
$$

Consider $B_{d, \mathcal{S}}$ in (36) with $U$ a constant. Expanding $Y_{d}$ using (2), we have

$$
\begin{aligned}
R_{\mathcal{S}} \leq C\left(\sum_{k \in \mathcal{S}} P_{k} / N_{d}+2 E[\right. & \left.X_{k} \tilde{X}_{r, \mathcal{S}}\right] / N_{d} \\
& \left.+E\left[\operatorname{var}\left(X_{r} \mid X_{\mathcal{S}^{c}}\right)\right] / N_{d}\right) .
\end{aligned}
$$


Using (5), (91), and (89), we simplify (94) as

$$
R_{\mathcal{S}} \leq C\left(\frac{\sum_{k \in \mathcal{S}} P_{k}+\bar{\gamma}_{\mathcal{S}^{c}} P_{r}+2 \sum_{k \in \mathcal{S}} \sqrt{\gamma_{k} P_{k} P_{r}}}{N_{d}}\right) .
$$

Writing $B_{r, \mathcal{S}}\left(\underline{\gamma}_{\mathcal{K}}\right)$ and $B_{d, \mathcal{S}}\left(\underline{\gamma}_{\mathcal{K}}\right)$ to denote the bounds on the right-hand side of (92) and (95), respectively, we have for a constant $U$, and a given choice of $\underline{\gamma}_{\mathcal{K}}$,

$$
R_{\mathcal{S}} \leq \min \left(B_{r, \mathcal{S}}\left(\underline{\gamma}_{\mathcal{K}}\right), B_{d, \mathcal{S}}\left(\underline{\gamma}_{\mathcal{K}}\right)\right), \quad \text { for all } \mathcal{S} \subseteq \mathcal{K}
$$

\section{APPENDIX II}

$$
\text { CONCAVITY OF } B_{d, \mathcal{S}}\left(\underline{\gamma}_{\mathcal{K}}\right) \text { AND } I_{d, \mathcal{S}}\left(\underline{\alpha}_{\mathcal{K}}, \underline{\beta}_{\mathcal{K}}\right)
$$

\section{A. Outer Bound $B_{d, \mathcal{S}}\left(\underline{\gamma}_{\mathcal{K}}\right)$}

Recall that the cutset bound at the destination, $B_{d, \mathcal{S}}\left(\underline{\gamma}_{\mathcal{K}}\right)$, is given by (17). We show that $B_{d, \mathcal{S}}\left(\underline{\gamma}_{\mathcal{K}}\right)$ is a concave function of $\underline{\gamma}_{\mathcal{K}}$. To prove concavity, one has to show that the Hessian or second derivative of $B_{d, \mathcal{S}}\left(\underline{\gamma}_{\mathcal{K}}\right), \nabla^{2} B_{d, \mathcal{S}}\left(\underline{\gamma}_{\mathcal{K}}\right)$, is negative semidefinite, i.e., $\underline{x}^{T} \nabla^{2} B_{d, \mathcal{S}}\left(\underline{\gamma}_{\mathcal{K}}\right) \underline{x} \leq 0$ for all $\underline{x} \in \mathcal{R}^{K}[24$, Sec. 3.1.4]. We write

$$
B_{d, \mathcal{S}}\left(\underline{\gamma}_{\mathcal{K}}\right)=\frac{1}{2} \log \left(K_{0}+2 \sum_{k \in \mathcal{S}} K_{k} \sqrt{\gamma_{k}}\right)
$$

where

$$
\begin{aligned}
& K_{0}=1+\frac{\sum_{k \in \mathcal{S}} P_{k}}{N_{d}}+\frac{P_{r}(1-c)}{N_{d}} \\
& K_{k}=\sqrt{\frac{P_{k}}{N_{d}} \frac{P_{r}}{N_{d}}}, \quad k \in \mathcal{S} .
\end{aligned}
$$

The gradient $\nabla B_{d, \mathcal{S}}$ is given by

$$
\begin{aligned}
\nabla B_{d, \mathcal{S}}\left(\underline{\gamma}_{\mathcal{K}}\right) & =\left[\partial B_{d, \mathcal{S}} / \partial \gamma_{k}\right]_{k \in \mathcal{K}} \\
& =\frac{1}{K_{s}}\left[\begin{array}{ll}
\underline{v}_{\mathcal{S}} & \underline{v}_{\mathcal{S}} c
\end{array}\right]^{T} \\
& =\frac{1}{K_{s}}
\end{aligned}
$$

where $\underline{v}_{\mathcal{S}}$ is an $|\mathcal{S}|$-length vector with entries $v_{k}=K_{k} / \sqrt{\gamma_{k}}$ for all $k \in \mathcal{S}, \underline{v}_{\mathcal{S}^{c}}$ is an $\left|\mathcal{S}^{c}\right|$-length vector with entries $v_{m}=$ $-P_{r} / N_{d}$ for all $m \in \mathcal{S}^{c}$, and

$$
K_{s}=2\left(K_{0}+2 \sum_{k \in \mathcal{S}} K_{k} \sqrt{\gamma_{k}}\right) \text {. }
$$

The Hessian of $B_{d, \mathcal{S}}\left(\underline{\gamma}_{\mathcal{K}}\right), \nabla^{2} B_{d, \mathcal{S}}\left(\underline{\gamma}_{\mathcal{K}}\right)$, is given by

$$
\begin{aligned}
\nabla^{2} B_{d, \mathcal{S}}\left(\underline{\gamma}_{\mathcal{K}}\right) & =\left[\partial^{2} B_{d, \mathcal{S}}\left(\underline{\gamma}_{\mathcal{K}}\right) / \partial \gamma_{k} \partial \gamma_{m}\right]_{\forall k, m \in \mathcal{K}} \\
& =\frac{-1}{K_{s}} \operatorname{diag}(\underline{d})-\underline{z z}^{T}
\end{aligned}
$$

where

$$
\begin{aligned}
& \underline{z}=\sqrt{2}\left(\nabla B_{d, \mathcal{S}}\left(\underline{\gamma}_{\mathcal{K}}\right)\right) \\
& \underline{d}=\left[\begin{array}{ll}
\underline{d}_{\mathcal{S}} & \underline{d}_{\mathcal{S}^{c}}
\end{array}\right]^{T}
\end{aligned}
$$

such that $\underline{d}_{\mathcal{S}}$ is an $|\mathcal{S}|$-length vector with entries $d_{k}=K_{k} / 2 \gamma_{k}^{3 / 2}$ for all $k \in \mathcal{S}$, and $\underline{d}_{\mathcal{S}^{c}}$ is an $\left|\mathcal{S}^{c}\right|$-length vector with entries $d_{k}=-2 P_{r}^{2} /\left(N_{d}^{2} K_{s}\right)$ for all $k \in \mathcal{S}^{c}$. Using the fact that $K_{k}$ and $\gamma_{k}$ are nonnegative for all $k$, from (98), for any $\underline{x} \in \mathcal{R}^{K}$, we have

$$
\underline{x}^{T} \nabla^{2} B_{d, \mathcal{S}}\left(\underline{\gamma}_{\mathcal{K}}\right) \underline{x}=-\frac{1}{K_{s}}\left(\sum_{k \in \mathcal{K}} x_{k}^{2} d_{k}\right)-\left(\underline{x}^{T} \cdot \underline{z}\right)^{2} \leq 0
$$

with equality if and only if $\underline{x}=\underline{0}$. In proving the concavity of $B_{d, \mathcal{S}}\left(\underline{\gamma}_{\mathcal{K}}\right)$, we assume only that $\gamma_{k}>0$, for all $k$. Thus, from continuity, the concavity also holds for all nonnegative $\gamma_{k}$ satisfying (see (12))

$$
\sum_{k \in \mathcal{K}} \gamma_{k} \leq 1
$$

Let $\underline{\gamma}_{\mathcal{A}}$ denote a vector with entries $\gamma_{k}$, for all $k \in \mathcal{A} \subseteq \mathcal{K}$. For a fixed $\underline{\gamma}_{\mathcal{S}}$, we now find the $\underline{\gamma}_{\mathcal{S}}$ that maximizes $B_{d, \mathcal{S}}\left(\underline{\gamma}_{\mathcal{K}}\right)$ subject to (99) above. For a $c \in[\overline{0}, 1)$, we fix $\underline{\gamma}_{\mathcal{S}^{c}}$ such that its entries $\gamma_{k}$, for all $k \in \mathcal{S}^{c}$, satisfy

$$
\sum_{k \in \mathcal{S}^{c}} \gamma_{k}=1-c
$$

and thus, from (99) we have

$$
\sum_{k \in \mathcal{S}} \gamma_{k} \leq c
$$

Since $B_{d, \mathcal{S}}\left(\underline{\gamma}_{\mathcal{K}}\right)$ is a continuous concave function of $\underline{\gamma}_{\mathcal{S}}$ it achieves its maximum at a $\gamma_{\mathcal{S}}^{*}$ where

$$
\left.\frac{\partial B_{d, \mathcal{S}}\left(\underline{\gamma}_{\mathcal{K}}\right)}{\partial \gamma_{k}}\right|_{\gamma_{k}^{*}}=0, \text { for all } k \in \mathcal{S} .
$$

Using the method of Lagrange multipliers, we find that for a fixed $\underline{\gamma}_{\mathcal{S}^{c}}$, the $\underline{\gamma}_{\mathcal{S}}$ that maximize $B_{d, \mathcal{S}}$ subject to (100) and (101) has entries given by

$$
\gamma_{k}^{*}=\left\{\frac{c P_{k}}{\sum_{k \in \mathcal{S}} P_{k}}, \quad k \in \mathcal{S} .\right.
$$

\section{B. Inner Bound $I_{d, \mathcal{S}}\left(\underline{\alpha}_{\mathcal{K}}, \underline{\beta}_{\mathcal{K}}\right)$}

Recall that the DF bound, $I_{d, \mathcal{S}}$, at the destination is given by (23). Comparing (17) and (23), for $\gamma_{k} \triangleq\left(1-\alpha_{k}\right) \beta_{k}$ for all $k \in \mathcal{S}$ and $\gamma_{k} \triangleq \beta_{k}$ for all $k \in \mathcal{S}^{c}$, the DF rate bound in (23) simplifies to that for the outer bound in (17), and thus, one can use the same technique to show that $I_{d, \mathcal{S}}\left(\underline{\alpha}_{\mathcal{K}}, \underline{\beta}_{\mathcal{K}}\right)$ is a concave function of $\underline{\alpha}_{\mathcal{K}}$ and $\underline{\beta}_{\mathcal{K}}$. For the power fractions $\beta_{k}$, we have

$$
\sum_{k \in \mathcal{K}} \beta_{k} \leq 1
$$

Let $\underline{\beta}_{\mathcal{A}}$ denote a vector with entries $\beta_{k}$, for all $k \in \mathcal{A} \subseteq$ $\mathcal{K}$. For a fixed $\underline{\alpha}_{\mathcal{K}}$, we determine the optimal $\underline{\beta}_{\mathcal{S}}$ maximizing $I_{d, \mathcal{S}}\left(\underline{\alpha}_{\mathcal{K}}, \underline{\beta}_{\mathcal{K}}\right)$ by fixing the vector $\underline{\beta}_{\mathcal{S}}$ such that

$$
\begin{aligned}
& \sum_{k \in \mathcal{S}^{c}} \beta_{k}=1-c \\
& \sum_{k \in \mathcal{S}} \beta_{k} \leq c
\end{aligned}
$$

where $c \in[0,1)$. Since $I_{d, \mathcal{S}}\left(\underline{\alpha}_{\mathcal{K}}, \underline{\beta}_{\mathcal{K}}\right)$ is independent of $\beta_{\mathcal{S}}$ for $\alpha_{\mathcal{S}}=1$, we assume that $\alpha_{\mathcal{S}} \neq 1$. 
We now consider the special case in which $\underline{\alpha}_{\mathcal{S}} \neq \underline{1}$ and $\underline{\beta}_{\mathcal{S c}}$ are fixed. We determine a $\underline{\beta}_{\mathcal{S}}$ that maximizes $I_{d, \mathcal{S}}\left(\underline{\alpha}_{\mathcal{K}}, \underline{\beta}_{\mathcal{K}}\right)$ subject to (104) and (103). Since $I_{d, \mathcal{S}}\left(\underline{\alpha}_{\mathcal{K}}, \beta_{\mathcal{K}}\right)$ is a continuous concave function of $\underline{\beta}_{\mathcal{S}}$, it achieves its maximum at a $\beta_{\mathcal{S}}^{*}$ where

$$
\left.\frac{\partial I_{d, \mathcal{S}}\left(\underline{\alpha}_{\mathcal{K}}, \underline{\beta}_{\mathcal{K}}\right)}{\partial \beta_{k}}\right|_{\beta_{k}^{*}}=0, \quad \text { for all } k \in \mathcal{S} .
$$

As before, using Lagrange multipliers, the optimal $\beta_{\mathcal{S}}^{*}$ that maximizes $I_{d, \mathcal{S}}\left(\underline{\alpha}_{\mathcal{K}}, \underline{\beta}_{\mathcal{K}}\right)$, subject to (104), has entries

$$
\beta_{k}^{*}=\left\{\frac{c\left(1-\alpha_{k}\right) P_{k}}{\sum_{k \in \mathcal{S}}\left(1-\alpha_{k}\right) P_{k}}, \quad k \in \mathcal{S} .\right.
$$

\section{APPENDIX III \\ $B_{r, \mathcal{S}}\left(\underline{\gamma}_{\mathcal{K}}\right)$ VERSUS $\gamma_{\mathcal{K}}$}

We show that the function $B_{r, \mathcal{S}}\left(\underline{\gamma}_{\mathcal{K}}\right)$ in (16) is a concave function of $\underline{\gamma}_{\mathcal{S}}$ for a fixed $\underline{\mathcal{S}}^{c}$ and for all $\mathcal{S} \subseteq \mathcal{K}$. Recall the expression for $B_{r, \mathcal{S}}\left(\underline{\gamma}_{\mathcal{K}}\right)$ as

$$
B_{r, \mathcal{S}}\left(\underline{\gamma}_{\mathcal{K}}\right)=C\left(\sum_{k \in \mathcal{S}} \frac{P_{k}}{N_{r}}-\frac{\left(\sum_{k \in \mathcal{S}} \sqrt{\gamma_{k} P_{k}}\right)^{2}}{N_{r}\left(1-\sum_{k \in \mathcal{S}^{c}} \gamma_{k}\right)}\right)
$$

where we assume that

$$
\sum_{k \in \mathcal{S}^{c}} \gamma_{k}=1-c<1
$$

Observe that $B_{r, \mathcal{S}}\left(\underline{\gamma}_{\mathcal{K}}\right)$ is maximized when $c=1$, i.e., $\gamma_{k}=0$ for all $k \in \mathcal{S}$, and minimized for $c=0$. Further, comparing $B_{r, \mathcal{S}}\left(\underline{\gamma}_{\mathcal{K}}\right)$ and $B_{d, \mathcal{S}}\left(\underline{\gamma}_{\mathcal{K}}\right)$, one can see that $B_{r, \mathcal{S}}\left(\underline{\gamma}_{\mathcal{K}}\right)$ achieves its minimum, i.e., $B_{r, \mathcal{S}}=0$, for

$$
\gamma_{k}= \begin{cases}P_{k} /\left(\sum_{k \in \mathcal{S}} P_{k}\right), & k \in \mathcal{S} \\ 0, & k \in \mathcal{S}^{c} .\end{cases}
$$

We write

$$
x \triangleq\left(\sum_{k \in \mathcal{S}} \sqrt{\gamma_{k} \lambda_{k}}\right)
$$

where

$$
P_{\max }=\max _{k \in \mathcal{K}} P_{k} \text { and } \lambda_{k}=P_{k} / P_{\max } .
$$

Substituting (107) in the expression for $B_{r, \mathcal{S}}\left(\underline{\gamma}_{\mathcal{K}}\right)$ in (106), we have

$$
B_{r, \mathcal{S}}\left(\underline{\gamma}_{\mathcal{K}}\right)=C\left(\sum_{k \in \mathcal{S}} \frac{P_{k}}{N_{r}}-\frac{x^{2} P_{\max }}{N_{r} c}\right) .
$$

Differentiating $B_{r, \mathcal{S}}\left(\underline{\gamma}_{\mathcal{K}}\right)$ with respect to $x$ we have

$$
\begin{aligned}
\frac{d B_{r, \mathcal{S}}\left(\underline{\gamma}_{\mathcal{K}}\right)}{d x} & =\frac{-x P_{\max }}{N_{r} c} \cdot\left(1+\sum_{k \in \mathcal{S}} \frac{P_{k}}{N_{r}}-\frac{x^{2} P_{\max }}{N_{r} c}\right)^{-1} \\
\frac{d^{2} B_{r, \mathcal{S}}\left(\underline{\gamma}_{\mathcal{K}}\right)}{d x^{2}} & =\frac{-\frac{P_{\max }}{N_{r} c}\left(1+\sum_{k \in \mathcal{S}} \frac{P_{k}}{N_{r}}\right)-\left(\frac{x P_{\max }}{N_{r} c}\right)^{2}}{\left(1+\sum_{k \in \mathcal{S}} \frac{P_{k}}{N_{r}}-\frac{x^{2} P_{\max }}{N_{r} c}\right)^{2}} \\
& <0
\end{aligned}
$$

where the strict inequality in (111) follows since all terms in (110) are positive. Further, for any $c>0$, from (109), $B_{r, \mathcal{S}}\left(\underline{\gamma}_{\mathcal{K}}\right)$ is maximized at $x=0$, i.e., for $\gamma_{k}=0$ for all $k \in \mathcal{S}$. Thus, we see that $B_{r, \mathcal{S}}\left(\underline{\gamma}_{\mathcal{K}}\right)$ is a concave decreasing function of $x$.

\section{APPENDIX IV \\ PROOF OF THEOREM 4}

We now prove Theorem 4 and give the solution to the maxmin optimization

$$
R_{\mathcal{K}}=\max _{\left(\underline{\alpha}_{\mathcal{K}}, \underline{\beta}_{\mathcal{K}}\right) \in \Gamma} \min \left(I_{r, \mathcal{K}}\left(\underline{\alpha}_{\mathcal{K}}\right), I_{d, \mathcal{K}}\left(\underline{\alpha}_{\mathcal{K}}, \underline{\beta}_{\mathcal{K}}\right)\right) .
$$

Consider the function

$$
J\left(\underline{\gamma}_{\mathcal{K}}, \delta\right)=\delta I_{r, \mathcal{K}}\left(\underline{\alpha}_{\mathcal{K}}\right)+(1-\delta) I_{d, \mathcal{K}}\left(\underline{\alpha}_{\mathcal{K}}, \underline{\beta}_{\mathcal{K}}\right), \quad \delta \in[0,1] .
$$

Observe that $J$ is linear in $\delta$ ranging in value from $I_{d, \mathcal{K}}\left(\underline{\alpha}_{\mathcal{K}}, \underline{\beta}_{\mathcal{K}}\right)$ for $\delta=0$ to $I_{r, \mathcal{K}}\left(\underline{\alpha}_{\mathcal{K}}\right)$ for $\delta=1$. Thus, the optimization in (113) is equivalent to maximizing the minimum of the two endpoints of the line $J$ over $\Gamma$. Maximizing $J\left(\underline{\alpha}_{\mathcal{K}}, \underline{\beta}_{\mathcal{K}}, \delta\right)$ over $\left(\underline{\alpha}_{\mathcal{K}}, \underline{\beta}_{\mathcal{K}}\right)$, we obtain a continuous convex function

$$
V(\delta)=\max _{\left(\underline{\alpha}_{\mathcal{K}}, \underline{\beta}_{\mathcal{K}}\right) \in \Gamma} J\left(\underline{\alpha}_{\mathcal{K}}, \underline{\beta}_{\mathcal{K}}, \delta\right), \quad \delta \in[0,1] .
$$

From (113) and (114), we see that for any $\left(\underline{\alpha}_{\mathcal{K}}, \underline{\beta}_{\mathcal{K}}\right)$, $J\left(\underline{\alpha}_{\mathcal{K}}, \underline{\beta}_{\mathcal{K}}, \delta\right)$ either lies strictly below or is tangential to $V(\delta)$. The following proposition summarizes a well-known solution to the max-min problem in (112) (see [9]).

Proposition 3: $\left(\underline{\alpha}_{\mathcal{K}, \delta}^{*}, \underline{\beta}_{\mathcal{K}, \delta}^{*}\right)$ is a max-min rule where

$$
\delta^{*}=\arg \min _{\delta \in[0,1]} V(\delta) .
$$

The maximum bound on $R_{\mathcal{K}}, V\left(\delta^{*}\right)$, is completely determined by the following three cases (see Fig. 3):

Case 1: $\quad \delta^{*}=0: V\left(\delta^{*}\right)=I_{r, \mathcal{K}}\left(\underline{\alpha}_{\mathcal{K}, \delta^{*}}^{*}\right)$

$$
<I_{d, \mathcal{K}}\left(\underline{\alpha}_{\mathcal{K}, \delta^{*}}^{*}, \underline{\beta}_{\mathcal{K}, \delta^{*}}^{*}\right)
$$

Case 2: $\quad 0<\delta^{*}<1: V\left(\delta^{*}\right)=I_{r, \mathcal{K}}\left(\underline{\alpha}_{\mathcal{K}, \delta^{*}}^{*}\right)$

$$
=I_{d, \mathcal{K}}\left(\underline{\alpha}_{\mathcal{K}, \delta^{*}}^{*}, \underline{\beta}_{\mathcal{K}, \delta^{*}}^{*}\right)
$$

Case 3: $\quad \delta^{*}=1: V\left(\delta^{*}\right)=I_{r, \mathcal{K}}\left(\underline{\alpha}_{\mathcal{K}, \delta^{*}}^{*}\right)$

$$
>I_{d, \mathcal{K}}\left(\underline{\alpha}_{\mathcal{K}, \delta^{*}}^{*}, \underline{\beta}_{\mathcal{K}, \delta^{*}}^{*}\right) .
$$

We apply Proposition 3 to determine the maximum bound on $R_{\mathcal{K}}$. We study each case separately and determine the max-min rule $\left(\underline{\alpha}_{\mathcal{K}, \delta^{*}}^{*}, \underline{\beta}_{\mathcal{K}, \delta^{*}}^{*}\right)$ for each case. In general, the max-min rule $\left(\underline{\alpha}_{\mathcal{K}, \delta^{*}}^{*}, \underline{\beta}_{\mathcal{K}, \delta^{*}}^{*}\right)$ depends on an optimal $\delta^{*}$. However, for notational convenience we henceforth omit the subscript $\delta^{*}$ in denoting the max-min rule. We develop the optimal $\left(\underline{\alpha}_{\mathcal{K}}^{*}, \underline{\beta}_{\mathcal{K}}^{*}\right)$ and the maximum sum-rate for each case. We first consider Case 3 and show that this case is not feasible.

We develop the conditions and determine the max-min rule for each case. 
Case 1: Consider the condition for Case 1 in (115). This condition implies that the case occurs when the maximum bound achievable at the relay is smaller than the bound at the destination. From (22), we observe that $I_{r, \mathcal{K}}$ increases monotonically with $\alpha_{k}$ for all $k$ and achieves a maximum of

$$
I_{r, \mathcal{K}}\left(\underline{\alpha}_{\mathcal{K}}^{*}\right)=C\left(\frac{\sum_{k \in \mathcal{K}} P_{k}}{N_{r}}\right)
$$

at $\underline{\alpha}_{\mathcal{K}}^{*}=\underline{1}$. Comparing (22) and (23) at $\underline{\alpha}_{\mathcal{K}}^{*}=\underline{1}$, we obtain the condition for this case as

$$
\frac{\sum_{k \in \mathcal{K}} P_{k}}{N_{r}} \leq \frac{\sum_{k \in \mathcal{K}} P_{k}+P_{r}}{N_{d}} .
$$

Case 2: Next we consider Case 2 in (116). This case occurs when the maximum rate bound achievable at the relay and destination are equal. The max-min solution for this case is obtained by considering two subcases. The first is the relatively straightforward subcase where $\underline{\alpha}_{\mathcal{K}}^{*}=\underline{1}$ is the max-min rule. The resulting maximum sum-rate is the same as that for Case 1 with (118) satisfied with equality. Consider the second subcase, where $\underline{\alpha}_{\mathcal{K}}^{*} \neq \underline{1}$, i.e.,

$$
\frac{\sum_{k \in \mathcal{K}} P_{k}}{N_{r}}>\frac{\sum_{k \in \mathcal{K}} P_{k}+P_{r}}{N_{d}} .
$$

In Appendix II, we show that, for a fixed $\alpha_{\mathcal{K}}, I_{d, \mathcal{S}}$, is a concave function of $\beta_{\mathcal{K}}$ for all $\mathcal{S} \subseteq \mathcal{K}$. Furthermore, from (19), for $\alpha_{\mathcal{K}} \neq$ $1, I_{d, \mathcal{K}}$ in (23) is maximized by a $\underline{\beta}_{\mathcal{K}}^{*}$ whose entries $\beta_{k}^{*}$, for all $k \in \mathcal{K}$, satisfy

$$
\sum_{k \in \mathcal{K}} \beta_{k}^{*}=1
$$

and are given by (28). Observe in (28) that the optimal power fraction $\beta_{k}^{*}$ that the relay allocates to cooperating with user $k$ is proportional to the power allocated by user $k$ to achieve coherent combining gains at the destination. Thus, one can formulate the optimization problem for this case as

$$
\begin{array}{ll}
\operatorname{maximize} & I_{r, \mathcal{K}}(\underline{\alpha}) \\
\text { subject to } & I_{r, \mathcal{K}}(\underline{\alpha})=I_{d, \mathcal{K}}(\underline{\alpha}, \underline{\beta}), \sum_{k \in \mathcal{K}} \beta_{k}=1 .
\end{array}
$$

Using Lagrange multipliers we can show that it suffices to con$\operatorname{sider} \beta_{k}=\beta_{k}^{*}$ in the maximization. Since the optimal $\beta_{k}^{*}$ in (28) is a function of $\alpha_{\mathcal{K}}, I_{d, \mathcal{K}}\left(\underline{\alpha}_{\mathcal{K}}, \underline{\beta}_{\mathcal{K}}^{*}\right)$ simplifies to a function of $\underline{\alpha}_{\mathcal{K}}$ as

$$
I_{d, \mathcal{K}}\left(\underline{\alpha}_{\mathcal{K}}, \underline{\beta}_{\mathcal{K}}^{*}\right)=C\left(\frac{\sum_{k \in \mathcal{K}} P_{k}+P_{r}+2 \sqrt{\sum_{k \in \mathcal{K}}\left(1-\alpha_{k}\right) P_{k} P_{r}}}{N_{d}}\right) .
$$

We further simplify $I_{d, \mathcal{K}}\left(\underline{\alpha}_{\mathcal{K}}, \underline{\beta}_{\mathcal{K}}^{*}\right)$ and $I_{r, \mathcal{K}}\left(\underline{\alpha}_{\mathcal{K}}\right)$ as follows. Choosing $P_{\max }$ and $\lambda_{k}$ as in (33), and for $q$ defined in (29), we obtain

$$
I_{r, \mathcal{K}}(q)=C\left(\left(\sum_{k \in \mathcal{K}} P_{k} / N_{r}\right)-q^{2} P_{\max } / N_{r}\right) \text { and }
$$

$$
I_{d, \mathcal{K}}(q)=C\left(\frac{\left(\sum_{k \in \mathcal{K}} P_{k}\right)+P_{r}+2 q \sqrt{P_{\max } P_{r}}}{N_{d}}\right) .
$$

Observe that $I_{r, \mathcal{K}}(q)$ and $I_{d, \mathcal{K}}(q)$ are monotonically increasing and decreasing functions of $q$ and thus, the maximization in (121) simplifies to determining a $q$ such that

$$
\frac{\sum_{k \in \mathcal{K}} P_{k}-q^{2} P_{\max }}{N_{r}}=\frac{\sum_{k \in \mathcal{K}} P_{k}+P_{r}+2 q \sqrt{P_{\max } P_{r}}}{N_{d}} .
$$

We can further simplify (122) using the definitions for the signal and noise power in (31). From (31), since $K_{3}>K_{2}$, (122) has only one positive solution given by

$$
q^{*}=\frac{-K_{1}+\sqrt{K_{1}^{2}+\left(K_{3}-K_{2}\right) K_{0}}}{K_{0}} .
$$

The max-min rules for this case is then the set $\mathcal{P}$ of $\left(\underline{\alpha}_{\mathcal{K}}^{*}, \underline{\beta}_{\mathcal{K}}^{*}\right)$ such that $\underline{\alpha}_{\mathcal{K}}^{*}$ satisfies (29) for $q=q^{*}$ and for each such choice of $\underline{\alpha}_{\mathcal{K}}^{*}, \underline{\beta}_{\mathcal{K}}^{*}$ is given by (28). The maximum achievable sum-rate for this case is then given by

$$
C\left(\frac{\left(\sum_{k \in \mathcal{K}} P_{k}\right)-\left(q^{*}\right)^{2} P_{\max }}{N_{r}}\right) .
$$

Case 3: Finally, we consider Case 3. This case occurs when the maximum bound achievable at the destination is smaller than the bound at the relay. Observe that $I_{d, \mathcal{K}}\left(\underline{\alpha}_{\mathcal{K}}, \underline{\beta}_{\mathcal{K}}\right)$ in (23) decreases monotonically with $\alpha_{k}$, for all $k$, and, for any $\beta_{\mathcal{K}}$, achieves a maximum at $\alpha_{\mathcal{K}}^{*}=0$ of

$$
I_{d, \mathcal{K}}\left(\underline{\alpha}_{\mathcal{K}}^{*}, \underline{\beta}_{\mathcal{K}}\right)=C\left(\frac{\sum_{k \in \mathcal{K}} P_{k}+P_{r}+2 \sum_{k \in \mathcal{K}} \sqrt{\beta_{k} P_{k} P_{r}}}{N_{d}}\right) .
$$

However, substituting $\alpha_{\mathcal{K}}^{*}=0$ in (22), we obtain

$$
I_{r, \mathcal{K}}\left(\underline{\alpha}_{\mathcal{K}}^{*}\right)=0
$$

which contradicts the assumption in (117), thus making this case infeasible.

\section{APPENDIX V}

\section{SUM-CAPACITY PROOF FOR THE ACTIVE ClasS}

In Theorem 7, we proved that DF achieves the sum-capacity for an active class of degraded Gaussian MARCs. In this proof we argue that since the maximum DF sum-rate is the same as the maximum outer bound sum-rate, every DF max-min rule $\left(\underline{\alpha}_{\mathcal{K}}^{*}, \underline{\beta}_{\mathcal{K}}^{*}\right) \in \mathcal{P}_{a}$ that achieves this maximum sum-rate, i.e., for which $R_{r}\left(\underline{\alpha}_{\mathcal{K}}^{*}\right) \cap R_{d}\left(\underline{\alpha}_{\mathcal{K}}^{*}, \underline{\beta}_{\mathcal{K}}^{*}\right)$ is an active case, also achieves the sum-capacity. We now present a more detailed proof of the argument.

We begin by comparing the inner and outer bounds. As in the symmetric case, without loss of generality, we write

$$
\gamma_{k}=\left(1-\alpha_{k}\right) \beta_{k}, \quad \text { for all } k
$$


where $\left(\underline{\alpha}_{\mathcal{K}}, \underline{\beta}_{\mathcal{K}}\right) \in \Gamma$. We then have

$$
B_{r, \mathcal{K}}\left(\underline{\alpha}_{\mathcal{K}}, \underline{\beta}_{\mathcal{K}}\right)=C\left(\sum_{k \in \mathcal{K}} \frac{P_{k}}{N_{r}}-\frac{\left(\sum_{k \in \mathcal{K}} \sqrt{\left(1-\alpha_{k}\right) \beta_{k} P_{k}}\right)^{2}}{N_{r}}\right) .
$$

On can verify in a straightforward manner that

$$
B_{d, \mathcal{K}}\left(\underline{\alpha}_{\mathcal{K}}, \underline{\beta}_{\mathcal{K}}\right)=I_{d, \mathcal{K}}\left(\underline{\alpha}_{\mathcal{K}}, \underline{\beta}_{\mathcal{K}}\right) .
$$

Choosing $\underline{\beta}_{\mathcal{K}}$ as the DF max-min rule $\underline{\beta}_{\mathcal{K}}^{*}$ in (28) simplifies (127) to

$$
B_{r, \mathcal{K}}\left(\underline{\alpha}_{\mathcal{K}}, \underline{\beta}_{\mathcal{K}}^{*}\right)=C\left(\sum_{k \in \mathcal{K}} \frac{\alpha_{k} P_{k}}{N_{r}}\right)=I_{r, \mathcal{K}}\left(\underline{\alpha}_{\mathcal{K}}\right) .
$$

Using Theorem 4 , one can then verify that $B_{r, \mathcal{K}}\left(\underline{\alpha}_{\mathcal{K}}^{*}, \underline{\beta}_{\mathcal{K}}^{*}\right)=$ $B_{d, \mathcal{K}}\left(\underline{\alpha}_{\mathcal{K}}^{*}, \underline{\beta}_{\mathcal{K}}^{*}\right)$ is achieved by all $\underline{\alpha}_{\mathcal{K}}^{*} \in \mathcal{P}$. Consider a $\underline{\alpha}_{\mathcal{K}}^{*} \in$ $\mathcal{P}_{a}$ and a corresponding $\beta_{\mathcal{K}}^{*}$ such that the DF region $\mathcal{R}_{r}\left(\underline{\alpha}_{\mathcal{K}}^{*}\right) \cap$ $\mathcal{R}_{d}\left(\underline{\alpha}_{\mathcal{K}}^{*}, \underline{\beta}_{\mathcal{K}}^{*}\right)$ is an active case. From Theorem 4 , this implies that

$$
I_{d, \mathcal{A}}\left(\underline{\alpha}_{\mathcal{K}}^{*}, \underline{\beta}_{\mathcal{K}}^{*}\right)+I_{r, \mathcal{A}^{c}}\left(\underline{\alpha}_{\mathcal{K}}^{*}\right)>I^{*}=B^{*}, \quad \text { for all } \mathcal{A} \subset \mathcal{K} .
$$

Using (126), we expand $B_{d, \mathcal{S}}$ in (17) as a function of $\left(\underline{\alpha}_{\mathcal{K}}^{*}, \underline{\beta}_{\mathcal{K}}^{*}\right)$ as

$$
\begin{gathered}
B_{d, \mathcal{S}}\left(\underline{\alpha}_{\mathcal{K}}^{*}, \underline{\beta}_{\mathcal{K}}^{*}\right)=C\left(\frac{\sum_{k \in \mathcal{K}} P_{k}+\left(1-\sum_{k \in \mathcal{S}^{c}}\left(1-\alpha_{k}^{*}\right) \beta_{k}^{*}\right) P_{r}}{N_{d}}\right. \\
\left.\frac{+2 \sum_{k \in \mathcal{S}} \sqrt{\left(1-\alpha_{k}^{*}\right) \beta_{k}^{*} P_{k} P_{r}}}{N_{d}}\right) \\
\geq I_{d, \mathcal{S}}\left(\underline{\alpha}_{\mathcal{K}}^{*}, \underline{\beta}_{\mathcal{K}}^{*}\right)
\end{gathered}
$$

where (130) follows from the fact that $\left(1-\alpha_{k}^{*}\right) \beta_{k}^{*} \leq \beta_{k}^{*}$, for all $k$ and for all $\left(\underline{\alpha}_{\mathcal{K}}^{*}, \underline{\beta}_{\mathcal{K}}^{*}\right)$. It is, however, not easy to compare $B_{r, \mathcal{S}}\left(\underline{\alpha}_{\mathcal{K}}^{*}, \underline{\beta}_{\mathcal{K}}^{*}\right)$ with $I_{r, \mathcal{S}}\left(\underline{\alpha}_{\mathcal{K}}^{*}\right)$. Note, however, that the choice of $\gamma_{k}$ in (126) requires the same source-relay correlation values for both the inner and outer bounds. Furthermore, for every choice of Gaussian input distribution with the same $K$ correlation values for both bounds, comparing the degraded cutset and DF bounds in (36) and (37), respectively, for a constant $U$, we have

$$
I\left(X_{\mathcal{S}} ; Y_{r} \mid X_{\mathcal{S}^{c}} X_{r}\right) \geq I\left(X_{\mathcal{S}} ; Y_{r} \mid X_{\mathcal{S}^{c}} V_{\mathcal{K}} X_{r}\right), \quad \text { for all } \mathcal{S} \subseteq \mathcal{K}
$$

where in (131) we use the fact that conditioning does not increase entropy to show that the cutset bounds at the relay are less restrictive than the corresponding DF bounds. From (128), the inequality in (131) simplifies to an equality for $\mathcal{S}=\mathcal{K}$ and for $\left(\underline{\alpha}_{\mathcal{K}}^{*}, \underline{\beta}_{\mathcal{K}}^{*}\right) \in \mathcal{P}_{a}$ when $\gamma_{k}$ is given by (126). Combining these inequalities with (129), we then have

$$
B_{d, \mathcal{A}}\left(\underline{\alpha}_{\mathcal{K}}^{*}, \underline{\beta}_{\mathcal{K}}^{*}\right)+B_{r, \mathcal{A}^{c}}\left(\underline{\alpha}_{\mathcal{K}}^{*}, \underline{\beta}_{\mathcal{K}}^{*}\right)>I^{*}=B^{*}, \quad \text { for all } \mathcal{A} \subset \mathcal{K} .
$$

Thus, every DF max-min rule that results in an active case polymatroid intersection, i.e., every $\left(\underline{\alpha}_{\mathcal{K}}^{*}, \underline{\beta}_{\mathcal{K}}^{*}\right) \in \mathcal{P}_{a}$ also results in an active case for the outer bounds when $\gamma_{k}$ is given by (126).

\section{ACKNOWLEDGMENT}

The authors wish thank Gerhard Kramer of Bell Labs, Alcatel-Lucent for numerous detailed discussions on the MARC, and Jan Vondrak of Princeton University for discussions on polymatroid intersections.

\section{REFERENCES}

[1] G. Kramer and A. J. van Wijngaarden, "On the white Gaussian multiple-access relay channel," in Proc. 2000 IEEE Int. Symp. Information Theory, Sorrento, Italy, Jun. 2000, p. 40.

[2] E. C. van der Meulen, "Three-terminal communication channels," $A d v$. Appl. Probab., vol. 3, pp. 120-154, 1971.

[3] T. Cover and A. El Gamal, "Capacity theorems for the relay channel," IEEE Trans. Inf. Theory, vol. IT-25, no. 5, pp. 572-584, Sep. 1979.

[4] G. Kramer, M. Gastpar, and P. Gupta, "Cooperative strategies and capacity theorems for relay networks," IEEE Trans. Inf. Theory, vol. 51, no. 9, pp. 3027-3063, Sep. 2005.

[5] L. Sankaranarayanan, G. Kramer, and N. B. Mandayam, "Capacity theorems for the multiple-access relay channel," in Proc. 42nd Annu. Allerton Conf. Communications, Control, and Computing, Monticello, IL, Sep. 2004, pp. 1782-1791.

[6] A. Reznik, S. R. Kulkarni, and S. Verdú, "Capacity and optimal resource allocation in the degraded Gaussian relay channel with multiple relays," IEEE Trans. Inf. Theory, vol. 50, no. 12, pp. 3037-3046, Dec. 2004

[7] L.-L. Xie and P. R. Kumar, "An achievable rate for the multiple-level relay channel," IEEE Trans. Inf. Theory, vol. 51, no. 4, pp. 1348-1358, Apr. 2005.

[8] A. El Gamal and M. Aref, "The capacity of the semideterministic relay channel," IEEE Trans. Inf. Theory, vol. IT-28, no. 3, p. 536, May 1982.

[9] Y. Liang, V. Veeravalli, and H. V. Poor, "Resource allocation for wireless fading relay channels: Max-min solution," IEEE Trans. Inf. Theory, vol. 53, no. 10, pp. 3432-3453, Oct. 2007.

[10] A. El Gamal and S. Zahedi, "Capacity of relay channels with orthogonal components," IEEE Trans. Inf. Theory, vol. 51, no. 5, pp. 1815-1817, May 2005.

[11] A. El Gamal and N. Hassanpour, "Capacity theorems for the relaywithout-delay channels," in Proc. 43nd Annu. Allerton Conf. Соттиnications, Control, and Computing, Monticello, IL, Sep. 2005.

[12] E. C. van der Meulen and P. Vanroose, "The capacity of a relay channel both with and without delay," IEEE Trans. Inf. Theory, vol. 53, no. 10, pp. 3774-3776, Oct. 2007

[13] T. M. Cover and J. A. Thomas, Elements of Information Theory. New York: Wiley, 1991.

[14] L. Sankaranarayanan, G. Kramer, and N. B. Mandayam, "Hierarchical sensor networks: Capacity theorems and cooperative strategies using the multiple-access relay channel model," in Proc. 1st IEEE Conf. Sensor and Ad Hoc Communications and Networks, Santa Clara, CA, Oct. 2004.

[15] A. Schrijver, Combinatorial Optimization: Polyhedra and Efficiency. New York: Springer-Verlag, 2003.

[16] R. G. Gallager, Information Theory and Reliable Communication. New York: Wiley, 1968.

[17] T. S. Han, "The capacity region of general multiple-access channel with certain correlated sources," Inf. Contr., vol. 40, no. 1, pp. 37-60, Jan. 1979.

[18] D. N. C. Tse and S. V. Hanly, "Multiaccess fading channels-Part I: Polymatroid structure, optimal resource allocation and throughput capacities," IEEE Trans. Inf. Theory, vol. 44, no. 7, pp. 2796-2815, Nov. 1998.

[19] L. Sankar, Y. Liang, N. B. Mandayam, and H. V. Poor, "Opportunistic Communications in Fading Multi-Access Relay Channels," 2009 [Online]. Available: arxiv.org e-print 0902.1220

[20] L. Sankar, G. Kramer, and N. B. Mandayam, "Offset encoding for multiple-access relay channels," IEEE Trans. Inf. Theory, vol. 53, no. 10, pp. 3814-3821, Oct. 2007. 
[21] H. G. Eggleston, Convexity. Cambridge, U.K.: Cambridge Univ. Press, 1958.

[22] H. V. Poor, An Introduction to Signal Detection and Estimation, 2nd ed. New York: Springer-Verlag, 1994.

[23] J. A. Thomas, "Feedback can at most double Gaussian multiple access channel capacity," IEEE Trans. Inf. Theory, vol. 33, no. 5, pp. 711-716, Sep. 1987.

[24] S. Boyd and L. Vandenberghe, Convex Optimization. Cambridge, U.K.: Cambridge Univ. Press, 2004.

Lalitha Sankar (S'92-M'07) received the B.Tech degree from the Indian Institute of Technology, Bombay, the M.S. degree from the University of Maryland, College Park, and the Ph.D. degree from Rutgers University, New Brunswick, NJ, in 2007.

Prior to her doctoral studies, she was a Senior Member of Technical Staff at AT\&T Shannon Laboratories. She is currently a Science and Technology Postdoctoral Fellow at Princeton University, Preinceton, NJ. Her research interests include wireless communications, network information theory, and information privacy and secrecy.

For her doctoral work, Dr. Sankar received the 2007-2008 Electrical Engineering Academic Achievement Award from Rutgers University.

Narayan B. Mandayam (S'90-M'95-SM'00-F'09) received the B.Tech (Hons.) degree in 1989 from the Indian Institute of Technology, Kharagpur, and the M.S. and Ph.D. degrees in 1991 and 1994 from Rice University, Houston, TX, all in electrical engineering.

From 1994 to 1996, he was a Research Associate at the Wireless Information Network Laboratory (WINLAB), Rutgers University, North Brunswick, NJ, before joining the faculty of the Electrical and Computer Engineering Department at Rutgers University, Piscataway, NJ, where he became Associate Professor in 2001 and Professor in 2003. Currently, he also serves as Associate Director at WINLAB. He was a visiting faculty fellow in the Department of Electrical Engineering, Princeton University, Princeton, NJ, in 2002 and a visiting faculty at the Indian Institute of Science in 2003. His research interests are in various aspects of wireless data transmission including system modeling and performance, signal processing and radio resource management with emphasis on techniques for cognitive radio networks. He is a coauthor with C. Comaniciu and H. V.
Poor of the book Wireless Networks: Multiuser Detection in Cross-Layer Design (New York: Springer-Verlag, 2005)

Dr. Mandayam is a recipient of the Fred W. Ellersick Prize from the IEEE Communications Society in 2009 along with O. Ileri for their work on dynamic spectrum access models and spectrum policy. He is also a recipient of the Institute Silver Medal from the Indian Institute of Technology in 1989 and the National Science Foundation CAREER Award in 1998. He has served as an Editor for the journals IEEE COMMUNICATION LETTERS and IEEE TRANSACTIONS ON WIRELESS COMMUNICATIONS. He has also served as a Guest Editor of the IEEE JouRnAl ON SELECTED AREAS IN COMMUNICATION Special Issues on Adaptive, Spectrum Agile and Cognitive Radio Networks (2007) and Game Theory in Communication Systems (2008).

H. Vincent Poor (S'72-M'77-SM' 82-F' 87) received the Ph.D. degree in electrical engineering and computer science from Princeton University, Princeton, $\mathrm{NJ}$, in 1977.

From 1977 until 1990, he was on the faculty of the University of Illinois at Urbana-Champaign, Urbana, IL. Since 1990, he has been on the faculty at Princeton University, where he is the Dean of Engineering and Applied Science, and the Michael Henry Strater University Professor of Electrical Engineering. His research interests are in the areas of stochastic analysis, statistical signal processing, and information theory, and their applications in wireless networks and related fields. Among his publications in these areas are Quickest Detection (Cambridge University Press, 2009), coauthored with Olympia Hadjiliadis, and Information Theoretic Security (Now Publishers, 2009), coauthored with Yingbin Liang and Shlomo Shamai.

Dr. Poor is a member of the National Academy of Engineering, a Fellow of the American Academy of Arts and Sciences, and an International Fellow of the Royal Academy of Engineering (U.K.). He is also a Fellow of the Institute of Mathematical Statistics, the Optical Society of America, and other organizations. In 1990, he served as President of the IEEE Information Theory Society, in 2004-2007 as the Editor-in-Chief of these TRANSACTIONS, and recently as General Co-Chair of the 2009 IEEE International Symposium on Information Theory, held earlier this year in Seoul, Korea. He is the recipient of the 2005 IEEE Education Medal. Recent recognition of his work includes the 2007 IEEE Marconi Prize Paper Award, the 2007 Technical Achievement Award of the IEEE Signal Processing Society, and the 2008 Aaron D. Wyner Distinguished Service Award of the IEEE Information Theory Society. 